\title{
Group-based Hierarchical Adaptive Traffic-Signal Control Part II: Implementation
}

\author{
Seunghyeon Lee ${ }^{1}$, S.C. Wong $^{2}$, Pravin Varaiya ${ }^{3}$ \\ ${ }^{1}$ Department of Civil and Natural Resources Engineering, University of Canterbury, Christchurch, \\ New Zealand \\ ${ }^{2}$ Department of Civil Engineering, the University of Hong Kong, Pokfulam Road, Hong Kong, China \\ ${ }^{3}$ Department of Electrical Engineering and Computer Sciences, University of California, Berkeley, \\ Berkeley, CA 94720-1722, USA
}

\begin{abstract}
In part I of this study (Lee et al. 2017), the formulation of a theoretical framework for a group-based adaptive traffic-control method for isolated signalized junctions is presented, which includes tactical and local levels of signal timing optimization. The global level control aims to determine the time-varying cycle structure, with a resolution of cycles, and the real-time adjustment of the green phase, with a resolution of seconds, based on longer-term traffic information observed by traffic detectors. Overall, the purpose of the study is to actualize a multi-resolution strategy for a group-based adaptive signal-control method and establish a microscopic simulation platform to implement the proposed methodology and test its effectiveness. To actualize the global proactiveoptimization scheme, in this paper, a rolling-horizon approach to the temporal and spatial variables, signal structures for four-arm intersections, and discrete directional search methods is applied using the developed mathematical framework. The formulation of the group-based max-pressure policy is realized using the logical form of the local reactive-control policy at a typical directional three-lane, four-arm approach to an isolated intersection. The integrated group-based adaptive traffic-signal control is actualized using VISSIM, Fortran, and VBA based on the developed tactical and local levels of signal timing optimization. The results of the computer simulations and the case study presented in this paper show that the integrated group-based adaptive traffic-signal-control logic outperforms the other methods over a wide range of traffic conditions, from free-flowing traffic to extreme congestion. Moreover, the proposed models perform much better than the existing fixedsignal plan and the actuated signal-control in asymmetric traffic conditions.
\end{abstract}

Keywords: Global proactive-optimization scheme, Local reactive-control policy, Simulation platform, Mathematical programming approach, Rolling-horizon approach

\section{INTRODUCTION}

In this paper, a multi-resolution strategy for the development of a group-based adaptive control method is proposed, in which a hierarchical structure consisting of two levels of signal control is designed, one for the global and one for the local level of signal timing optimization presented in Lee et al. (2017). The global level control aims to determine the time-varying cycle structure, with a resolution of cycles, and the real-time adjustment of the green phase, with a resolution of seconds, based on longer-term traffic information as observed by traffic detectors. The global level uses a group-based optimization method to determine the cycle-structure based on the traffic information from the previous cycles with a resolution of minutes. Finally, the local level uses a reactive localcontrol logic to update the stage-switching times based on local traffic information with a resolution 
of seconds. The proposed multi-resolution strategy serves to simultaneously determine the global signal settings and update the local signal parameters in a unified and systematic framework that can help improve the performance of signalized isolated junctions effectively and efficiently. The objective of the papers is to actualize a multi-resolution strategy for a group-based adaptive signalcontrol method and establish a microscopic simulation platform to implement the proposed methodology and test its effectiveness.

A proactive global-optimization procedure is developed for group-based signal settings at the global level of control. According to the real-time queue-length estimation process explained in Lee et al. (2015a), the prevalent arrival rates are obtained for the individual lanes approaching a signalized junction so that the patterns of arrival at the junction can be discerned. An arrival pattern is defined as the pattern of traffic that would arrive at the stop line at the end of a lane if the traffic were not impeded by the signals at the stop line. As shown in Wong (1995), the queue-formation pattern is classified into several types, based on the set of analytical expressions that are derived for estimating the derivatives of the queuing delay with respect to a change in the signal timing. The procedure for using queue-formation patterns is explored in Lee and Wong (2017a, 2017b). Using this procedure, the total delay at the junction for a given signal plan and its derivatives are estimated with respect to the changes in timings, which serve as the objective function and the gradient information for the formulation of a mathematical program for conducting the proactive global-optimization procedure, as developed in Lee et al. (2017). In this mathematical program, the group-based timing variables, i.e., the start and the duration of the green phase for each signal group and the successor functions between conflicting signal groups, are defined as control variables. The set of constraints includes the constraints on the duration of the green signal, the clearance time, the capacity, and the various other constraints that govern the additional safety and operational requirements specified by the users. The process of optimizing the application of these control and constraint variables is clarified in this paper.

The advantage of the group-based optimization method is that the most effective cycle structure (or the best overall pattern of signal stages) can be obtained automatically. The definitions of the stages, and the combinations, sequences, and structures of the inter-green periods between consecutive stages, do not need to be pre-specified. This freedom from pre-determined controls allows a high degree of flexibility in tactically identifying the optimized signal plan in response to the realtime-traffic information. However, despite these advantages, and given that the group-based optimization procedure is highly efficient (Wong 1996, 1997), it is anticipated that a certain amount of computing time will still be required to obtain the optimized solution. Therefore, the global approach is used to compute the group-based solution during the current cycle and to implement this optimized solution in the next cycle, which helps to provide a window for the computational work. Nevertheless, the signal control in the current cycle is adjusted reactively in real time by the local level of control. The resolution of this global control level is in minutes because the cycle length and cycle-structures are allowed to vary from cycle to cycle.

Based on the global level of control, the cycle length and the cycle structure of the signal plan for a junction are obtained before the commencement of the current cycle. Nevertheless, traffic may still vary locally, and can deviate from the patterns used for computation at the global level. Therefore, a reactive local signal-control policy is developed to locally adjust the signal timings in response to the second-by-second variations in traffic patterns, as based on the max-pressure signal-control policy (Varaiya 2013). From the global control plan, the group-based optimization procedure identifies the most appropriate cycle-structure according to the latest available information for which the number of stages, sequences, and durations have been generated automatically. At the local level of control, a transition zone is set before and after each switching time from one stage to the next (e.g., three 
seconds for each zone). During the real-time operation of the current cycle, the queuing information is monitored from the real-time, second-by-second queue-length estimation procedure, as developed in Lee et al. (2015a). The trigger switching then occurs in the next stage, when the criteria specified by the local control policy are met or when the time involved reaches the end of the transition zone. Therefore, the stage-switching time can occur before or after the time set by the global plan. The resolution of this local control level is in seconds, because changes to the signal features are decided second-by-second.

The use of a simulation platform is one of the possible alternatives for evaluating and verifying the performance of the newly developed traffic-control algorithms. The main advantage of this approach is that it helps to generate identical inputs for the evaluation of different algorithms, while avoiding the high installation and operational costs involved in a real-traffic system. This approach is also free from the safety concerns relating to tests that involve conflicting traffic movements during the development stage. Traffic, signal, and geometrical information is collected for the target intersection, and is implemented using the VISSIM (Verkehr In Staedten SIMulation) microscopic simulation platform. Fortran programming language is used to code the proactive global group-based signal-control algorithm to optimize the signal timings cycle-by-cycle at the global level. The visual basic application (VBA) is used to install the algorithm that performs the real-time groupbased adjustments of each green phase second-by-second at the local level and sets the responsive time frames for the analysis and transition zones. VBA is also used to construct the synthetic framework that connects the microscopic simulation program, mathematical program, local adjustments, and algorithms for the temporal frames. The performance of the proposed methods is compared with those of the existing fixed-control and actuated-control methods to evaluate the effectiveness of the developed methodology.

In the following section, the integrated framework of the adaptive traffic-signal-control logic is introduced. The implementation of the global proactive-optimization method, including a rollinghorizon approach, the plan for the signal structures and saturation flow rates, and a mathematical programming approach, is illustrated in Section 3. The implementation of the local reactive-control policy is described in Section 4. A set of numerical examples and a case study are given in Sections 5 and 6, respectively. Finally, conclusions and recommendations for further research are provided in Section 7.

\section{INTEGRATED ADAPTIVE TRAFFIC-SIGNAL-CONTROL LOGIC}

To integrate the global proactive-optimization procedure and the local reactive-control policy (the mathematical frameworks for these procedures are presented in Lee et al. (2017)), the performance of a typical directional three-lane, four-arm approach to an isolated intersection was simulated using the VISSIM computer software. The VISSIM COM interface was used to construct an overall recursive process for the global optimization of the signal timings and local adjustment of the green phases at the isolated signalized junction. To establish the framework of the recursive process for the control logic, VBA was used for the main algorithm and Fortran with IMSL was used to obtain the solutions for the linear and non-linear mathematical programming for the globaloptimization method. The framework of the simulation, including the cycle-by-cycle global optimization and the second-by-second local adjustment, is depicted in Figure 1. 


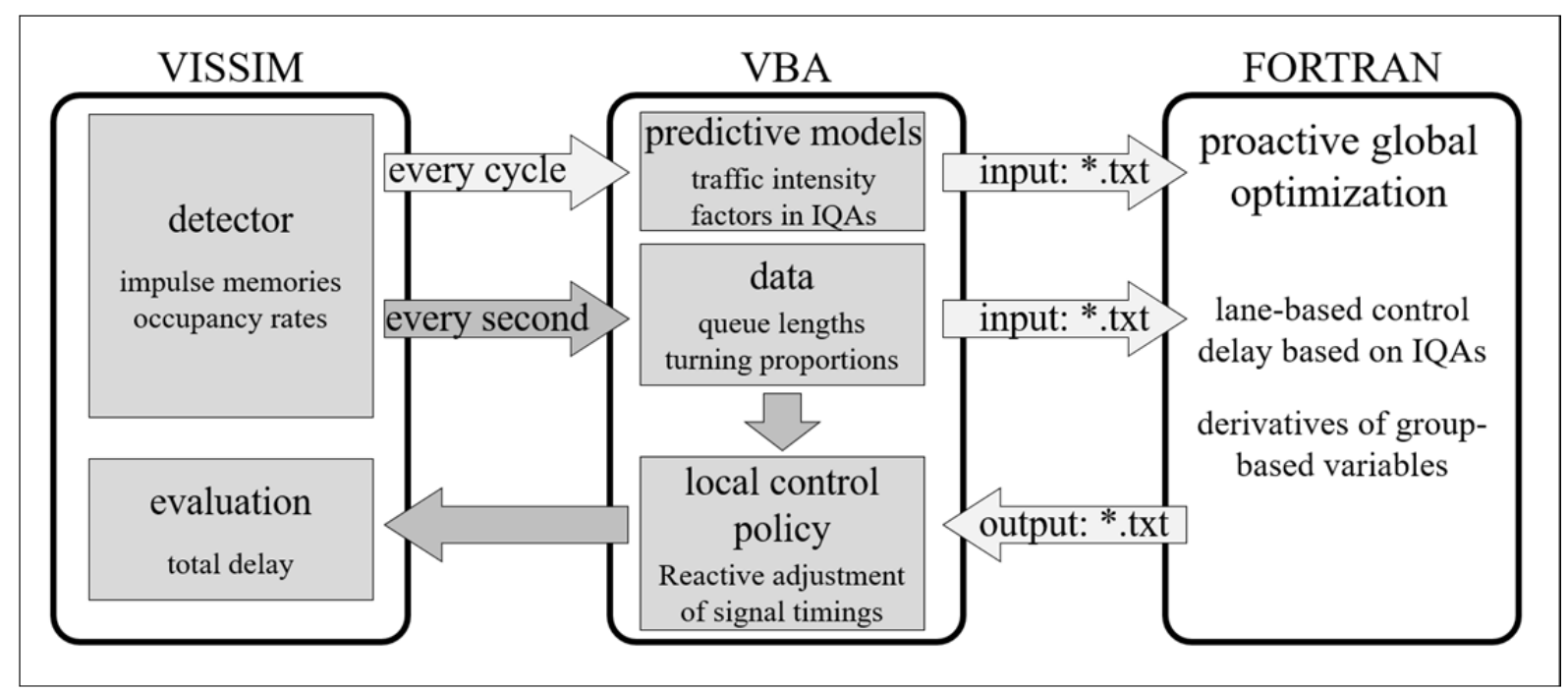

Figure 1. The framework of the integrated adaptive traffic-signal-control logic

As shown in Figure 1, the collected detector data on VISSIM are used to estimate the lane-based queue lengths and the lane-based turning proportions second-by-second. In addition, the estimated traffic information is applied to develop a lane-based formulation in VBA for the IQAs, adjustment factors, queue lengths at inflection points, and the traffic intensities for each cycle. The rollinghorizon approach to recursive processes for the control logic is used to predict the future traffic information. Then the optimized signal plan, including the start and duration of green time, the cycle time, and the signal sequence, is produced by the proactive global-optimization method coded in Fortran. The local reactive-control policy is applied within a transition zone based on the optimized signal plans in VBA and VISSIM to minimize the total delay as an evaluation step.

\section{GLOBAL PROACTIVE-OPTIMIZATION SCHEME}

The VISSIM COM interface, managed by VBA, is used to connect all of the modules for the data collection, estimation methods, predictive models, recursive processes, and optimization methods, which were developed in the previous studies of the authors. At the step of the data collection, the occupancy rates and impulse memories collected from the downstream and exit detectors on the previous cycle are used to calculate both the lane-based traffic volumes and the turning proportions. At the step of estimation methods, the estimated lane-based turning proportions are then used as input data to compute the lane-based saturation flows in relation to the geometric information on the target intersection. In addition, the lane-based queue lengths are estimated based on the occupancy rates and impulse memories, as collected from the downstream and upstream detectors. At the step of predictive models and recursive processes, the procedure for computing the IQAs and the temporal and spatial information on the lane-based queue lengths in the previous cycle is explained in Lee and Wong (2017b). The outputs of the estimation methods are used as the inputs for the lane-based rollinghorizon approach, which predicts the traffic flows, adjustment factors, and queue lengths at the inflection points on the IQAs for the next cycle. Finally, the predicted future traffic flows are used to establish the constraints for maximizing the reserve capacity. The final successor function and the initial guess for the nonlinear mathematical programs are achieved through maximizing the reserve capacity, and these outcomes are then used to minimize the control delay in the next cycle. Furthermore, the predicted formulations of the IQAs are established based on the predicted temporal and spatial information on the lane-based queue lengths, and these IQAs are used to construct the objective function and the gradient for minimizing the control delay developed in Lee and Wong 
(2017a). The traditional framework for optimizing the group-based variables and the results from all of the previous steps are used to minimize the control delay for the future cycle. The optimized groupbased variables, including the start points and durations of the green time for each signal group and the cycle time, serve to specify the signal plan for the next cycle. These three computational procedures are applied to the current cycle, based on the data collected from the previous cycle, to construct the signal structure for the next cycle. This data-processing method is performed in each cycle for each individual lane at the optimization methods.

Fortran with IMSL is used to identify the most appropriate successor functions under each given traffic circumstance, and to determine the initial guesses for the non-linear programs using the methods for maximizing the reserve capacity. In addition, the method for minimizing the control delay on the successive cycles provides the final group-based variables for the next cycle by using non-linear mathematical programs coded in Fortran with IMSL. To illustrate the proactive global group-based signal-control algorithm, which was introduced as the mathematical framework for global proactive-optimization scheme in Lee et al. (2017), the proposed framework of the simulation is described in Figure 2.

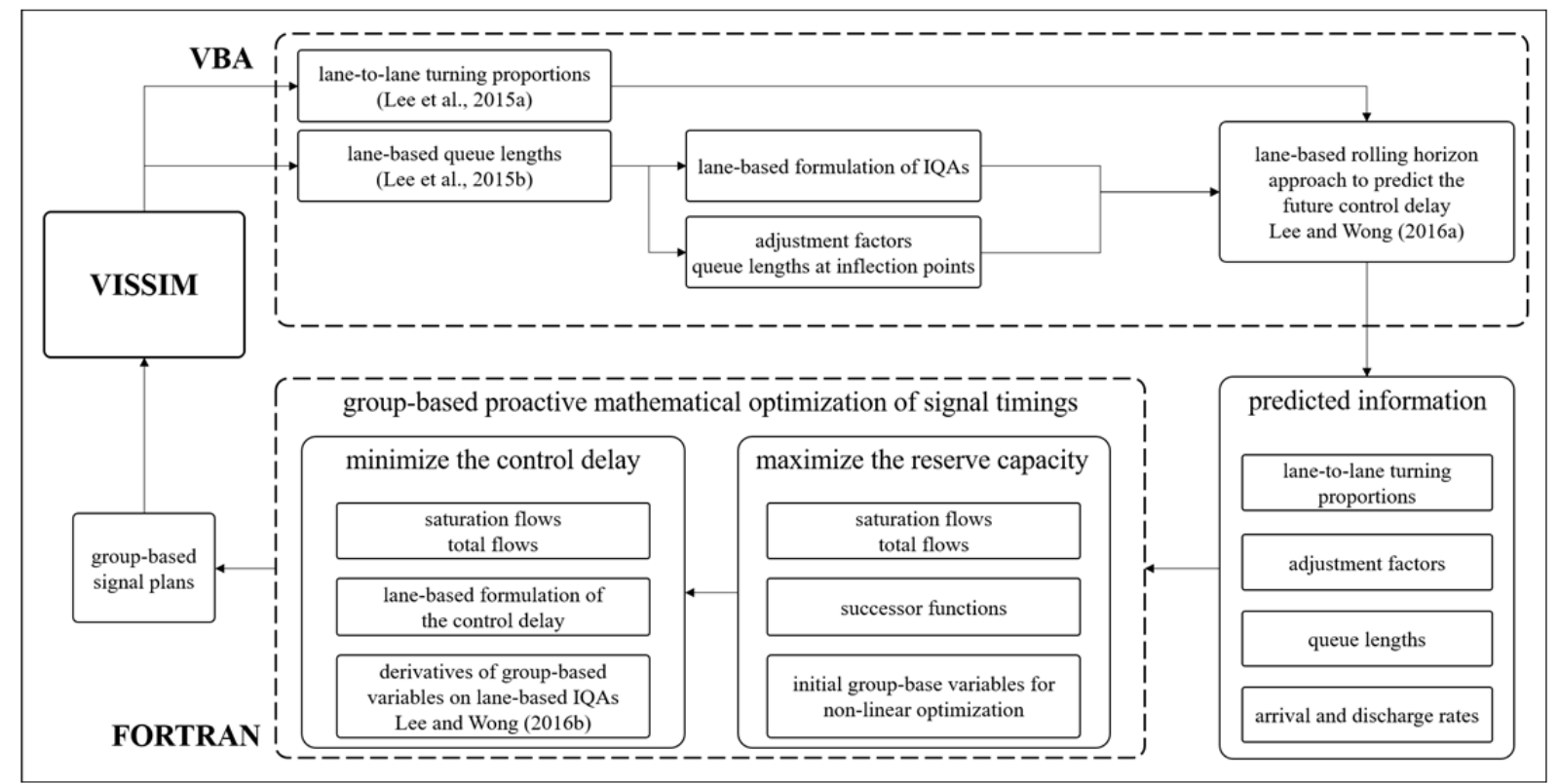

Figure 2. The framework of the simulation

As shown in Figure 2, the framework for the data processing is installed to run a simulation using VISSIM traffic simulation, VISSIM COM interface, VBA, and Fortran with IMSL. For the data collection, the impulse memories and occupancy rates are collected from the set of exit, downstream, and upstream detectors in real time. VBA is used to create the lane-to-lane turning proportions and queue lengths in the individual lanes (which are estimated by the methods developed in Lee et al. (2015b) and Lee et al. (2015a)) on the basis of the collected data set. In addition, the lane-based trapezoids and triangles in the IQAs (as developed in Lee and Wong (2017b)) on a cycle with adjustment factors and queue lengths at the inflection points are derived from the lane-based queue lengths estimated using the VBA codes. The data-processing method, in which the temporal and spatial information on the traffic conditions are used as the input data, follows the lane-based rollinghorizon approach developed in Lee and Wong (2017b). The predicted future temporal and spatial parameters are coded by VBA based on the VISSIM COM interface. Then, the set of predicted traffic information, including the lane-to-lane turning proportions, adjustment factors, queue lengths at the 
inflection points in individual lanes, and arrival and discharge rates, is used as input data to devise the group-based proactive global-optimization method, which is coded using Fortran with IMSL.

In the real-time group-based optimization procedure, the lane-to-lane turning proportions and arrival and discharge rates are used to calculate the traffic intensities. The traffic movements are evaluated with reference to the maximum acceptable levels of saturation in traffic flows and traffic volumes to solve the capacity maximization problem for each cycle. The most suitable successor function for the predicted traffic situation is selected according to the maximum values for the reserve capacities among the 32 sets of possible successor functions. The initial values of the group-based variables for the non-linear mathematical programs are also provided through the capacity maximization problem. Based on the output from the previous optimization process, a lane-based formulation to calculate the area of the polygons in the IQAs for the control delay is used as the objective function, as developed in Lee and Wong (2017b). Moreover, the approximate expressions of the derivatives, as created in Lee and Wong (2017a), are applied as gradients to identify a better local optimum through non-linear programming.

The group-based variables for minimizing the control delay on the future three cycles with respect to the given predicted traffic conditions are implemented for each next future cycle in VISSIM by using the VISSIM COM interface. The developed recursive process for identifying the optimum structure for the next cycle is performed in each cycle, and the sub-modules in the recursive process are connected by VBA.

\subsection{Rolling-horizon approach}

The rolling-horizon process, which is introduced in Lee and Wong (2017a) and based on the Kalman filter, is used to predict the traffic intensities and parameters of the delay formula. The prediction of future patterns, which is introduced in the data-processing method in Lee et al. (2017), is conducted based on the collected detector data from the previous cycle and the recursive process for predicting future traffic intensities, along with the temporal and spatial information for the control delays on the IQAs. The traffic intensities $v_{k}^{n}$ and $s_{k}^{n}$, the adjustment factors $\boldsymbol{\Omega}_{k}^{n}$, and the queue lengths at inflection points $\Psi_{k}^{n}$ for the future IQAs are all predicted using an effective and efficient recursive process of time-series analysis. For $\boldsymbol{\Omega}_{k}^{n}$ and $\Psi_{k}^{n}$, the recursive process is already illustrated by the time windows, as explained in the previous section, and the system variables, time-update equations, and measurement equations are set to predict $v_{k}^{n}$ and $s_{k}^{n}$. The set of traffic volumes at downstream detectors $v_{k}^{n-1}$ and the set of saturation flows $s_{k}^{n-1}$ in the $(n-1)$ th cycle are all defined as the measurement variables because the recursive procedures and optimization procedures are all performed in the current cycle, i.e., the $n$th cycle. For the future time-windows, $v_{k}^{n+x}$ and $s_{k}^{n+x}$ are defined as the state variables for cycle $n$ along with the variable $x$, which is the future cycle following the current $n$th cycle. The rolling-horizon process of using the Kalman filter to estimate $\hat{v}_{k}^{n+x}$ and $\hat{S}_{k}^{n+x}$ is described in Lee and Wong (2017b). This process is an abridged version of the process given by Lee et al. (2015a). $\hat{v}_{k}^{n+x}$ and $\hat{s}_{k}^{n+x}$ are then used to estimate the traffic intensities in the $(n+x)$ th cycle. The superscript $\left(^{-}\right)$indicates an a priori state estimate at step $i$, and a circumflex $\left(^{\wedge}\right)$ indicates an a posteriori estimate of the state at cycle $n$. The step of the recursive process is set as $i$. The rolling- 
horizon procedure for all of the time windows to predict the traffic intensities follows the process represented in Lee and Wong (2017b), instead of using $\boldsymbol{\Omega}_{k}^{n}$ and $\Psi_{k}^{n}$.

\subsection{Signal structures}

To confirm the effectiveness of the proposed formulation for a signal structure in Lee et al. (2017), all of the possible cycle-structures are categorized into 32 equivalence classes of successor functions, according to the Heydecker (1992) method, for a typical layout of an isolated signalized intersection with a directional three-lane and four-arm approach. The following figures illustrate the definitions and sets of successor functions to be considered.

Table 1. The directional three-lane and four-arm approach to a typical intersection

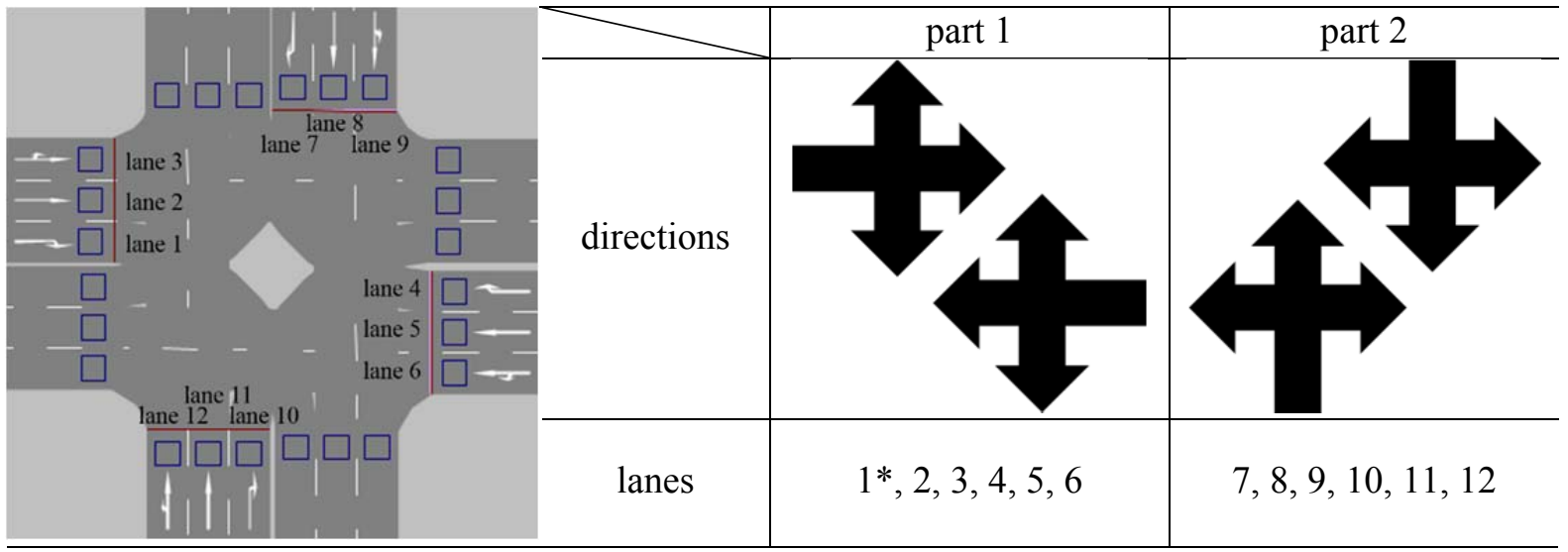

* the start signal group on each cycle

In Table 1, a typical layout of an isolated signalized junction is described with the compatible lane groups organized into two parts. The east- and west-bound traffic-stream lanes, comprising lanes $1,2,3,4,5$, and 6 , are set as the lane groups in part 1, and the north- and south-bound lanes, comprising lanes $7,8,9,10,11$, and 12 , are set as the lane groups in part 2 . Lane 1 is in the start signal group in each cycle, and all of the lanes within the same part are compatible, whereas they are not compatible with the lanes of the other part. For the part 1 lanes, the equivalence classes of successor functions are categorized in Table 2.

Table 2. Four equivalence classes of successor functions in part 1

\begin{tabular}{c|cccccc}
$k$ & & \multicolumn{7}{c}{$\mathbf{1}$} \\
$k$ & 1 & 2 & 3 & 4 & 5 & 6 \\
\hline 1 & 0 & 0 & 0 & 0 & 0 & 0 \\
2 & 0 & 0 & 0 & 0 & 0 & 0 \\
3 & 0 & 0 & 0 & 0 & 0 & 0 \\
4 & 1 & 1 & 1 & 0 & 0 & 0 \\
5 & 1 & 1 & 1 & 0 & 0 & 0 \\
6 & 1 & 1 & 1 & 0 & 0 & 0
\end{tabular}

\begin{tabular}{c|cccccc}
$k$ & 1 & 2 & 3 & 4 & 5 & 6 \\
\hline 1 & 0 & 0 & 0 & 0 & 0 & 0 \\
2 & 0 & 0 & 0 & 0 & 0 & 0 \\
3 & 0 & 0 & 0 & 0 & 0 & 0 \\
4 & 0 & 1 & 1 & 0 & 0 & 0 \\
5 & 1 & 0 & 0 & 0 & 0 & 0 \\
6 & 1 & 0 & 0 & 0 & 0 & 0
\end{tabular}

\begin{tabular}{c|llllll}
$k$ & 1 & 2 & 3 & 4 & 5 & 6 \\
\hline 1 & 0 & 0 & 0 & 0 & 0 & 0 \\
2 & 1 & 0 & 0 & 1 & 0 & 0 \\
3 & 1 & 0 & 0 & 1 & 0 & 0
\end{tabular}

\begin{tabular}{c|cccccc}
$k j$ & 1 & 2 & 3 & 4 & 5 & 6 \\
\hline 1 & 0 & 0 & 0 & 0 & 0 & 0 \\
2 & 0 & 0 & 0 & 1 & 0 & 0 \\
3 & 0 & 0 & 0 & 1 & 0 & 0
\end{tabular}




$$
\begin{array}{l|lllllll|llllll}
4 & 0 & 0 & 0 & 0 & 0 & 0 & 4 & 0 & 0 & 0 & 0 & 0 & 0 \\
5 & 1 & 0 & 0 & 1 & 0 & 0 & 5 & 1 & 0 & 0 & 0 & 0 & 0 \\
6 & 1 & 0 & 0 & 1 & 0 & 0 & 6 & 1 & 0 & 0 & 0 & 0 & 0
\end{array}
$$

In Table 2, the first matrix of the binary variables in a successor function indicates that all of the traffic streams on the east-bound lanes have the right-of-way first, and then a green signal is assigned to all of the traffic streams on the west-bound lanes. The second successor function illustrates that the green time for all of the traffic streams on the east-bound lanes is the first stage. Then, one of the east-bound traffic streams enters the inter-green phase, and incompatible traffic streams on the west-bound lanes (where the green time for the previous stage is finishing) have the right-of-way. Right-turn movements on both the east- and west-bound lanes have the right-of-way first on the third and fourth successor functions. Then, the green time for the passing-through and leftturn flows on both bounds is assigned to the third successor function, whereas one of the left-turn flows is overlapped with compatible movements on the fourth successor function. For the successor functions in the part 2 lanes, eight equivalence classes of successor functions are classified, as show in Table 3.

Table 3. Eight equivalence classes of successor functions in part 2

\begin{tabular}{|c|c|c|c|c|c|c|c|c|c|c|c|c|c|}
\hline & & & 1 & & & & & & & 2 & & & \\
\hline$k^{j}$ & 7 & 8 & 9 & 10 & 11 & 12 & $k$ & 7 & 8 & 9 & 10 & 11 & 12 \\
\hline 7 & 0 & 0 & 0 & 0 & 0 & 0 & 7 & 0 & 0 & 0 & 0 & 0 & 0 \\
\hline 8 & 0 & 0 & 0 & 0 & 0 & 0 & 8 & 0 & 0 & 0 & 0 & 0 & 0 \\
\hline 9 & 0 & 0 & 0 & 0 & 0 & 0 & 9 & 0 & 0 & 0 & 0 & 0 & 0 \\
\hline 10 & 1 & 1 & 1 & 0 & 0 & 0 & 10 & 0 & 1 & 1 & 0 & 0 & 0 \\
\hline 11 & 1 & 1 & 1 & 0 & 0 & 0 & 11 & 1 & 0 & 0 & 0 & 0 & 0 \\
\hline 12 & 1 & 1 & 1 & 0 & 0 & 0 & 12 & 1 & 0 & 0 & 0 & 0 & 0 \\
\hline & & & 3 & & & & & & & 4 & & & \\
\hline$k^{j}$ & 7 & 8 & 9 & 10 & 11 & 12 & & 7 & 8 & 9 & 10 & 11 & 12 \\
\hline 7 & 0 & 0 & 0 & 0 & 0 & 0 & 7 & 0 & 0 & 0 & 0 & 0 & 0 \\
\hline 8 & 1 & 0 & 0 & 1 & 0 & 0 & 8 & 0 & 0 & 0 & 1 & 0 & 0 \\
\hline 9 & 1 & 0 & 0 & 1 & 0 & 0 & 9 & 0 & 0 & 0 & 1 & 0 & 0 \\
\hline 10 & 0 & 0 & 0 & 0 & 0 & 0 & 10 & 0 & 0 & 0 & 0 & 0 & 0 \\
\hline 11 & 1 & 0 & 0 & 1 & 0 & 0 & 11 & 1 & 0 & 0 & 0 & 0 & 0 \\
\hline 12 & 1 & 0 & 0 & 1 & 0 & 0 & 12 & 1 & 0 & 0 & 0 & 0 & 0 \\
\hline & & & 5 & & & & & & & 6 & & & \\
\hline$k^{J}$ & 7 & 8 & 9 & 10 & 11 & 12 & $k$ & 7 & 8 & 9 & 10 & 11 & 12 \\
\hline 7 & 0 & 0 & 0 & 1 & 1 & 1 & 7 & 0 & 0 & 0 & 0 & 1 & 1 \\
\hline 8 & 0 & 0 & 0 & 1 & 1 & 1 & 8 & 0 & 0 & 0 & 1 & 0 & 0 \\
\hline 9 & 0 & 0 & 0 & 1 & 1 & 1 & 9 & 0 & 0 & 0 & 1 & 0 & 0 \\
\hline 10 & 0 & 0 & 0 & 0 & 0 & 0 & 10 & 0 & 0 & 0 & 0 & 0 & 0 \\
\hline 11 & 0 & 0 & 0 & 0 & 0 & 0 & 11 & 0 & 0 & 0 & 0 & 0 & 0 \\
\hline 12 & 0 & 0 & 0 & 0 & 0 & 0 & 12 & 0 & 0 & 0 & 0 & 0 & 0 \\
\hline & & & 7 & & & & & & & 8 & & & \\
\hline$k^{j}$ & 7 & 8 & 9 & 10 & 11 & 12 & $k$ & 7 & 8 & 9 & 10 & 11 & 12 \\
\hline 7 & 0 & 1 & 1 & 0 & 1 & 1 & 7 & 0 & 0 & 0 & 0 & 1 & 1 \\
\hline 8 & 0 & 0 & 0 & 0 & 0 & 0 & 8 & 0 & 0 & 0 & 0 & 0 & 0 \\
\hline
\end{tabular}




\begin{tabular}{c|ccccccc|cccccc}
9 & 0 & 0 & 0 & 0 & 0 & 0 & 9 & 0 & 0 & 0 & 0 & 0 & 0 \\
10 & 0 & 1 & 1 & 0 & 1 & 1 & 10 & 0 & 1 & 1 & 0 & 0 & 0 \\
11 & 0 & 0 & 0 & 0 & 0 & 0 & 11 & 0 & 0 & 0 & 0 & 0 & 0 \\
12 & 0 & 0 & 0 & 0 & 0 & 0 & 12 & 0 & 0 & 0 & 0 & 0 & 0
\end{tabular}

In Table 3, the successor functions are categorized into eight equivalence classes for the part 2 lanes, as for the part 1 lanes, except for the fixed starting signal group. Specifically, one of the traffic streams on the north-bound lanes is the first signal group from the first to the fourth successor functions, and one of the traffic streams on south-bound lanes has the first right-of-way from the fifth to the eighth successor functions in part 2. This pattern reflects the condition that the start signal group in part 2 is not constrained to be unchangeable. The two parts of the successor functions are combined based on the format shown in Table 4.

Table 4. The format of successor functions, including the intersection's two parts

\begin{tabular}{c|cccccc|cccccc}
$k$ & 1 & 2 & 3 & 4 & 5 & 6 & 7 & 8 & 9 & 10 & 11 & 12 \\
\hline 1 & & & & & & & 0 & 0 & 0 & 0 & 0 & 0 \\
2 & & & & & & & 0 & 0 & 0 & 0 & 0 & 0 \\
3 & & & & & & & 0 & 0 & 0 & 0 & 0 & 0 \\
4 & & & part 1 & & & 0 & 0 & 0 & 0 & 0 & 0 \\
5 & & & & & & & 0 & 0 & 0 & 0 & 0 & 0 \\
6 & & & & & & & 0 & 0 & 0 & 0 & 0 & 0 \\
\hline 7 & 1 & 1 & 1 & 1 & 1 & 1 & & & & & & \\
8 & 1 & 1 & 1 & 1 & 1 & 1 & & & & & & \\
9 & 1 & 1 & 1 & 1 & 1 & 1 & & & & & & \\
10 & 1 & 1 & 1 & 1 & 1 & 1 & & & & & \\
11 & 1 & 1 & 1 & 1 & 1 & 1 & & & & & &
\end{tabular}

In Table 4, the matrix of binary variables illustrates that part 1 precedes part 2 in each cycle. The above-mentioned equivalence classes for both parts are united, and there are 32 equivalence classes in this typical layout of an isolated signalized junction. A maximization procedure for determining the reserve capacity is performed 32 times on each cycle, and then the successor function $\hat{\Lambda}_{k j}^{n}$ (which identifies the greatest reserve capacity with the optimized group-based variables and the given traffic conditions during a cycle) is chosen to minimize the control delay in real time in the next step. The selected successor function is used for the constants on the left-hand side of the inequality constraints for the clearance time, which define a cycle-structure in terms of both capacity maximization and delay minimization.

\subsection{Saturation flow rates}

Saturation flow rates are used to calculate the traffic intensities of the inequality constraints on both capacity maximization and delay minimization, as developed in Section 4 in Lee et al. (2017), 
for the target intersection. Saturation flow rates are defined in HCM (2010) as "the flow, in vehicles per hour, that can be accommodated by the lane group, assuming that the green phases were displayed 100 percent of the time." The base saturation flow rates per lane $k$ on the $n$th cycle, and on $s_{k}^{n}$, which is an exclusive straight-ahead lane, are given by Equations (1) and (2), as presented in the Transport Planning and Design Manual (1986).

$s_{k}^{n}=1940+100\left(w_{k}-3.25\right)$ for nearside lane or single lane entries

$s_{k}^{n}=2080+100\left(w_{k}-3.25\right)$ for non-nearside lanes

where $w_{k}$ is the lane width at entry in meters.

For lanes with mixed traffic, the saturation flow rates are adjusted by Equation (3), as explained in the Transport Planning and Design Manual (1986).

$\widetilde{s}_{k}^{n}=\frac{s_{k}^{n}}{1+\frac{1.5}{R_{k}} f_{k}^{n}}$

In Equation (3), $s_{k}^{n}$ is the base saturation flow rate, and $R_{k}$ is a radius of the curvature of vehicle paths (m). $f_{k}^{n}$ is the left- or right-turning proportions in lane $k$ in the $n$th cycle, which is the output of the methods for estimating the lane-based turning proportions, as explained in Lee et al. (2017). The final adjusted saturation flows vary in each cycle, and are used to construct the constraints for the traffic intensities on the optimization procedures.

\subsection{Mathematical programming approach}

We used the discrete directional search method without gradients and Powell's TOLMIN in Fortran IMSL to solve the proposed linear problem for the maximization of the reserve capacity, and non-linear mathematical programs for the minimization of the control delay, respectively, in Lee et al. (2017). The mathematical programs to solve the given problems are designed in the Fortran Numerical Library, in which Fortran is connected with the VBA programs to perform the optimization procedures and run the recursive procedures, including the data collection and processing methods, respectively. The linear and non-linear programming approaches for solving the given mathematical problems in Lee et al. (2017) are provided in the following sub-sections.

\subsubsection{Linear programming approach for maximizing the reserve capacity}

The optimization problem for maximizing the reserve capacity can be formulated cycle-bycycle as in the following linear mathematical program:

$\max \mu^{n}$

subject to $A^{n}\left\{\begin{array}{l}\Gamma^{n} \\ \mu^{n}\end{array}\right\} \leq\left\{\begin{array}{c}0 \\ \Lambda^{n}\end{array}\right\}, \forall n \in N$ 
where $\mu^{n}$ is the value of the reserve capacity of the signal-controlled intersections when the signal timings are $\Gamma^{n}$, with the successor function $\boldsymbol{\Lambda}^{n}$, under the given constraint $A^{n}$, on the $n$th cycle. The comparison of the reserve capacities (which result from conducting the capacity maximization procedure 32 times on the basis of the differing $A^{n}$ and $\boldsymbol{\Lambda}^{n}$ values expressed in Equations (4) and (5)) is performed during the $(n-1)$ th cycle, based on data collected in the $(n-2)$ th cycle, to determine the initial guesses for the group-based variables and the most appropriate cycle-structure for the nonlinear mathematical programs on the $n$th cycle. The linear optimization procedure for maximizing the reserve capacity is established according to the discrete directional search method without gradients. The mathematical program for finding a solution is provided in the IMSL Fortran Numerical Library, namely the routine LCONF, which is similar to the routine LCONG as described in sub-section 3.4.2. The defined $\Gamma_{0}^{n}$ and $\hat{\Lambda}^{n}$ are transferred to the delay minimization procedure, which is explained in the next sub-section.

\subsubsection{Non-linear programming approach to minimizing the control delay}

The non-linear mathematical problems are solved by the routine LCONG, which is based on Powell's (1989) TOLMIN in Fortran. This routine features a non-linear objective function, various linear equality/inequality constraints, and a set of analytic gradients. The general mathematical formula, as provided by the IMSL Fortran Numerical Library, is as follows:

$$
\begin{aligned}
& \min _{x \in R^{n}}=f(t), \\
& \text { subject to } A_{1} x=b_{1}, A_{2} x \leq b_{2}, x_{l} \leq x \leq x_{u},
\end{aligned}
$$

where the vectors $b_{1}, b_{2}, x_{l}$, and $x_{u}$ are the right-hand-side vectors for the equality and inequality constraints and the upper and lower bounds of the variables, respectively, and where the matrices $A_{1}$ and $A_{2}$ are the left-hand-side vectors for the equality and inequality constraints, respectively. In this section, $x$ is the set of group-based variables for the future three cycles, $\bar{\Gamma}^{n} \supset \Gamma^{n}, \Gamma^{n+2}, \Gamma^{n+3}$, which are to be optimized in the non-linear mathematical programming process. The set of variables for the first cycle consists of $\Gamma^{n}=\left\{\zeta^{n}, \theta_{1}^{n} \ldots \theta_{k}^{n}, \phi_{1}^{n} \ldots \theta_{k}^{n}\right\}, \forall k \in K, \forall n \in N . A_{1}$ and $A_{2}$ are the vectors of the coefficients in the general constraints in the group-based optimization method for defining the signal structures and the sequence of the effective green phases in the future three cycles. These future cycles are decided on the previous step, namely the maximization of the reserve capacity. Corresponding to the vectors on the left-hand side, the successor function is included in $b_{1}$ and $b_{2}$ along with the zero column to define the inter-green structure of the given signal structure in the future three cycles.

The iteration process follows the routine LCONG entirely, as given in the IMSL Fortran Numerical Library. The inconsistency and redundancy in the equality constraints are examined in the first step of the algorithm. In equation (7), $x$ is the input variable at the $k$ th iteration for the iteration routine of the non-linear programming. If the equality constraints are consistent, then $x^{0}$ (which is the initial guess provided from the maximization of the reserve capacity) is revised to satisfy $A_{1} x=b_{1}$. Then the method adjusts $x^{0}$ to satisfy the simple bounds and the inequality constraints. A sequence of quadratic programming sub-problems solves the above-given algorithm to minimize the sum of the constraint or bound violations. For each iteration with a feasible $x^{k}$, let $J^{k}$ be the set of indices of the inequality constraints that have small residuals. The simple bounds are dealt with as inequality constraints. Let $I^{k}$ be the set of indices of the active constraints. The quadratic programming problem is as follows: 
$\min f\left(x^{k}\right)+d^{T} \nabla f\left(x^{k}\right)+\frac{1}{2} d^{T} B^{k} d$,

subject to $a_{j} d=0, j \in I_{k}$ and $a_{j} d \leq 0, j \in J_{k}$,

which is solved to find ( $d k, \lambda k$ ), where $a_{j}$ is a row vector in the constraints $A_{1}$ and $A_{2}$, and in the simple bound constraints on $x$. For the simple bound constraints, $a_{j}=e_{i}$ for the bound constraint $x_{i} \leq\left(x_{u}\right)_{i}$, and $a_{j}=-e_{i}$ for the constraint $-x_{i} \leq\left(-x_{u}\right)_{i}$. Then, $e_{i}$ is a vector having 1 as the $i$-th component, and 0 elsewhere. $\lambda k$ is the Lagrange multiplier, and $B^{k}$ is a positive definite approximation to the second set of derivatives, $\nabla^{2} f\left(x^{k}\right)$. After the search direction $d^{k}$ is obtained, a line search is performed to locate a better point. The new point $x^{k+1}=x^{k}+\alpha^{k} d^{k}$ has to satisfy the following conditions:

$f\left(x^{k}+\alpha^{k} d^{k}\right) \leq f\left(x^{k}\right)+0.1 \alpha^{k}\left(d^{k}\right)^{T} \nabla f\left(x^{k}\right)$

and

$\left(d^{k}\right)^{T} \nabla f\left(x^{k}+\alpha^{k} d^{k}\right) \geq 0.7\left(d^{k}\right)^{T} \nabla f\left(x^{k}\right)$.

The main idea in forming the set $J^{k}$ is that if any of the inequality constraints restrict the steplength $\alpha^{k}$, then its index is not in $J^{k}$. Therefore, small steps are likely to be avoided. Finally, the second derivative approximation, $B^{k}$, is updated by the BFGS formula, if the condition

$\left(d^{k}\right)^{T} \nabla f\left(x^{k}+\alpha^{k} d^{k}\right)-\nabla f\left(x^{k}\right)>0$

holds. Let $x^{k} \leftarrow x^{k+1}$, and start another iteration. The iteration repeats until the stopping criterion

$\left\|\nabla f\left(x^{k}\right)-A^{k} \lambda^{k}\right\|_{2} \leq \tau$

is satisfied. Here, $\tau$ is a user-supplied tolerance. For more details, see Powell (1989a, 1989b). This discrete directional search method is performed at the end of each cycle to find the optimized groupbased variables with the objective functions and gradients described below.

The IMSL Fortran Numerical Library, which is a widely used set of reliable, robust routines for mathematics and statistics, is used to search for the optimum values. The general form defined by the routine LCONG is described in the abovementioned mathematical procedure. Detailed explanations are provided in the User's Guide MATH/LIBRARY of the IMSL Fortran Numerical Library and in Powell (1989a, 1989b).

\section{LOCAL REACTIVE-CONTROL POLICY}

In this section, the logical formulation for the local reactive-control policy, developed in Lee et al. (2017), is realized by the detailed implementation plans and flow charts in the VBA computer program. The implementation plan establishes the integrated framework of group-based approach to the max-pressure control policy and transition zones for group-based variables.

\subsection{The implementation of the local reactive-control policy}

The transient and persistent variations in traffic flows are captured and handled by the developed local reactive-control policy and the global proactive-optimization method, respectively. To automatically adjust the current green time according to the pressure incurred by the current traffic conditions, a mathematical framework of the group-based max-pressure method (with temporal variables) is developed for the layout of a general signal-controlled intersection. This intersection includes four arms and three traffic movements for each arm, with a given condition that the first 
signal group is fixed in every cycle. A local clock for each signal group is provided to restrict the progression of each green time by imposing a transition zone (TZ), which is determined with reference to the group-based temporal variables. In addition, a state and a local clock for each signal group is defined according to the relevant segment of the timeline, divided by the transition zone. The detailed algorithm for this process is explained by the following set of conditions.

1) Signal group 1 , which can proceed from 0 up to the end of a transition zone $\left(0<t_{1}<\frac{\hat{\phi}_{1}^{n}}{\zeta_{c}^{n}}+\right.$ $\mathrm{TZ}$ ), is the first signal group in every cycle.

A. $t_{1}>0$, and $\left(t_{1} \leq \frac{\hat{\phi}_{1}^{n}}{\zeta_{c}^{n}}-\mathrm{TZ}\right.$, or $\left.t_{1} \leq 5\right)$

The states of a signal controller at $t_{1}=0$ are defined on the basis of successor functions. If $\Lambda_{2,4}^{n}=0$, then the states of the signal groups 1 and 2 are set as green, with the rest of the signal group set as red. Otherwise, groups 1 and 4 are set as green, with the rest as red. The local clock runs for the signal group (by which the green time is assigned), whereas the local clock is set as 0 for the amber and red times. Then, the assigned states are maintained as the minimum green time, or until the lower bound of a transition zone is reached.

B. $t_{1}>\frac{\hat{\phi}_{1}^{n}}{\zeta_{c}^{n}}-\mathrm{TZ}$, and $t_{1}>5$, and $t_{1}<\frac{\hat{\phi}_{1}^{n}}{\zeta_{c}^{n}}+\mathrm{TZ}$

The green time in signal group 1 can be terminated with the start of the green time in signal group 3 for each pair of incompatible signal groups, according to the pressure on each group in this section. If $\sum_{k=1}^{K_{1}} s_{k}^{n} q_{k}^{n}(t) \geq \sum_{k=1}^{K_{3}} s_{k}^{n} q_{k}^{n}(t)$, the current state of group 1 is constant. Otherwise, the state of group 1 is in the inter-green period.

C. $t_{1} \geq \frac{\hat{\phi}_{1}^{n}}{\zeta_{c}^{n}}+\mathrm{TZ}$

The green time in signal group 1 should be terminated at the start of the green time for the other signal group in each pair of incompatible signal groups at the upper bounds of a transition zone. Then, the state of group 1 is in the inter-green period.

2) Signal group 2 can either precede or follow its paired signal group 4, because these groups follow different rules according to the value of the successor function, $\Lambda_{2,4}^{n}$. Signal group 2 can proceed from 0 up to the end of a transition zone $\left(0<t_{2}<\frac{\hat{\phi}_{2}^{n}}{\zeta_{c}^{n}}+\mathrm{TZ}\right)$ regardless of the signal group pair's temporal position.

A. $t_{2}>0$, and $\left(t_{2} \leq \frac{\hat{\phi}_{2}^{n}}{\zeta_{c}^{n}}-\mathrm{TZ}\right.$, or $\left.t_{2} \leq 5\right)$

If $\Lambda_{2,4}^{n}=0$, the green time for signal group 2 starts with signal group 1 at the initial time in a cycle, and the green time for signal group 4 starts subsequent to that of signal group 2. Otherwise, groups 1 and 4 are set as green at the start of a cycle and signal 
group 2 follows group 4. Then, the assigned states are maintained as the minimum green, or until the lower bound of a transition zone is reached.

B. $t_{2}>\frac{\hat{\phi}_{2}^{n}}{\zeta_{c}^{n}}-\mathrm{TZ}$, and $t_{2}>5$, and $t_{2}<\frac{\hat{\phi}_{2}^{n}}{\zeta_{c}^{n}}+\mathrm{TZ}$

If $\Lambda_{2,4}^{n}=0$, the green time for signal group 2 can be terminated to begin the green time for signal group 4 , according to the pressure on each group in this period. If $\sum_{k=1}^{K_{2}} s_{k}^{n} q_{k}^{n}(t) \geq \sum_{k=1}^{K_{4}} s_{k}^{n} q_{k}^{n}(t)$, then the current state of group 2 is constant. Otherwise, the state of group 2 is in the inter-green period. However, if $\Lambda_{2,4}^{n}=1$, then the green time for signal groups 2 and 3 can be terminated to begin the green time for the next clique, according to the results of the comparison of the pressure on signal groups 2 and 3 and the pressure on the first signal groups in the following clique. The first signal groups in the following clique vary with the values of $\Lambda_{5,7}^{n}$ and $\Lambda_{6,8}^{n}$, in which four combinations of signal groups are considered. If the current states of the traffic flows satisfy Equation (46), the right-of-way in the signal controller is continuously assigned to signal groups 2 and 3. Otherwise, the current state of the signal controller is in the inter-green time.

C. $t_{2} \geq \frac{\hat{\phi}_{2}^{n}}{\zeta_{c}^{n}}+\mathrm{TZ}$

If $\Lambda_{2,4}^{n}=0$, then the green time for signal group 2 should be terminated to change the current state of group 4, and the state of group 2 is in the inter-green period. If $\Lambda_{2,4}^{n}=1$, then the green time for signal groups 2 and 3 is terminated, and the first signal groups in the next clique start after the inter-green time for those groups.

3) For signal group 3, which is definitely the last signal group in the pair of signal groups 1 and 3 , the assignment of green times follows relatively simple rules. Signal group 3 can proceed from 0 up to the end of a transition zone $\left(0<t_{3}<\frac{\hat{\phi}_{3}^{n}}{\zeta_{c}^{n}}+\mathrm{TZ}\right)$.

A. $t_{3}>0$, and $\left(t_{3} \leq \frac{\hat{\phi}_{3}^{n}}{\zeta_{c}^{n}}-\mathrm{TZ}\right.$, or $\left.t_{3} \leq 5\right)$

The state of signal group 3 is set as green, and the local clock of the signal group assigns the green time. Then, the assigned states are maintained as the minimum green, or until the lower bound of a transition zone is reached.

B. $t_{3}>\frac{\hat{\phi}_{3}^{n}}{\zeta_{c}^{n}}-\mathrm{TZ}$, and $t_{3}>5$, and $t_{3}<\frac{\hat{\phi}_{3}^{n}}{\zeta_{c}^{n}}+\mathrm{TZ}$

If $\Lambda_{2,4}^{n}=0$, then the green time for signal groups 3 and 4 can be terminated to start the green time for the first signal groups in the next clique, according to the pressure on these groups and the next signal groups. Otherwise, the green times for groups 2 and 3 can be terminated to begin the green time for the following signal groups. If the current situation of the traffic flows satisfies Equation (46), then the current state of the signal 
controller is continued. Otherwise, the state of the signal groups to which the green time is assigned changes to the inter-green period. The combinations of the first signal groups in the next clique depend on the values of the successor functions, $\Lambda_{5,7}^{n}$ and $\Lambda_{6,8}^{n}$.

C. $t_{3} \geq \frac{\hat{\phi}_{3}^{n}}{\zeta_{c}^{n}}+\mathrm{TZ}$

The current state of the signal controller is immediately terminated, and the green time of the current signal groups is changed to the inter-green time. Then, the first signal groups in the next clique, as defined by the successor functions $\Lambda_{5,7}^{n}$ and $\Lambda_{6,8}^{n}$, have a right-of-way after the inter-green period.

4) Signal group 4, which can either precede or follow its paired signal group 2, follows rules similar to those of signal group 2, according to the value of the successor function, $\Lambda_{2,4}^{n}$. Signal group 4 can proceed from 0 up to the end of a transition zone $\left(0<t_{4}<\frac{\hat{\phi}_{4}^{n}}{\zeta_{c}^{n}}+\mathrm{TZ}\right)$, regardless of the temporal position of the pair.

A. $t_{4}>0$, and $\left(t_{4} \leq \frac{\hat{\phi}_{4}^{n}}{\zeta_{c}^{n}}-\mathrm{TZ}\right.$ or $\left.t_{4} \leq 5\right)$

If $\Lambda_{2,4}^{n}=1$, the green time is assigned to signal group 4 at the initial time in a cycle, and the green time for signal group 2 starts subsequent to that for signal group 4. Otherwise, groups 1 and 2 are set as green at the start of a cycle, and signal group 4 follows group 2 . Then, the assigned states are maintained as the minimum green, or maintained until the lower bound of a transition zone is reached.

B. $t_{4}>\frac{\hat{\phi}_{4}^{n}}{\zeta_{c}^{n}}-\mathrm{TZ}$, and $t_{4}>5$, and $t_{4}<\frac{\hat{\phi}_{4}^{n}}{\zeta_{c}^{n}}+\mathrm{TZ}$

If $\Lambda_{2,4}^{n}=1$, then the green time for signal group 4 can be terminated to begin the green time for signal group 2, according to the pressure on each group in this period. If $\sum_{k=1}^{K_{4}} s_{k}^{n} q_{k}^{n}(t) \geq \sum_{k=1}^{K_{2}} s_{k}^{n} q_{k}^{n}(t)$, then the current state of group 4 is constant. Otherwise, the state of group 4 is in the inter-green period. However, if $\Lambda_{2,4}^{n}=0$, then the green time for signal groups 3 and 4 can be terminated to begin the green time for the next clique, according to the comparison of the pressure on signal groups 3 and 4 and that on the first signal groups in the following clique. Determining the first signal groups in the following clique depends on the values of $\Lambda_{5,7}^{n}$ and $\Lambda_{6,8}^{n}$, which involve four combinations of signal groups. If the current states of traffic flows satisfy Equation (46), then the right-of-way in the signal controller is continuously assigned to signal groups 3 and 4. Otherwise, the current state of the signal controller is in the inter-green time.

C. $t_{4} \geq \frac{\hat{\phi}_{4}^{n}}{\zeta_{c}^{n}}+\mathrm{TZ}$ 
If $\Lambda_{2,4}^{n}=1$, then the green time for signal group 4 should be terminated to change the current state of group 2, and the state of group 4 is in the inter-green period. If $\Lambda_{2,4}^{n}=0$, then the green time for signal groups 3 and 4 is terminated, and the first signal groups in the next clique start after the inter-green time for those groups.

5) Signal groups 5, 6, 7, and 8 in the second clique, which can either precede or follow each other in their paired signal groups (of 5 and 7, and of 6 and 8), all follow similar rules according to the binary values of the successor functions $\Lambda_{5,7}^{n}$ and $\Lambda_{6,8}^{n}$. The possible combinations of the preceding signal groups are (SG5, SG6), (SG5, SG8), (SG7, SG6), and (SG7, SG8), which are derived from $(0,0),(0,1),(1,0)$, and $(1,1)$, as $\left(\Lambda_{5,7}^{n}, \Lambda_{6,8}^{n}\right)$, respectively. The possible combinations of the following signal groups are (SG5, SG6), (SG5, SG8), (SG7, SG6), and (SG7, SG8), which are derived from $(1,1),(1,0),(0,1)$, and $(0,0)$, as $\left(\Lambda_{5,7}^{n}, \Lambda_{6,8}^{n}\right)$, respectively. The index for signal groups $5,6,7$, and 8 is set as $x$ for designating the variables and parameters of these signal groups, except for their successor functions. In addition, the index of the signal groups that are paired with signal groups $5,6,7$, and 8 , is set as $y$. These signal groups can proceed from 0 up to the end of a transition zone $\left(0<t_{x}<\frac{\hat{\phi}_{x}^{n}}{\zeta_{c}^{n}}\right.$ $+\mathrm{TZ}$ ), regardless of the temporal position of the pair.

A. $t_{x}>0$, and $\left(t_{x} \leq \frac{\hat{\phi}_{x}^{n}}{\zeta_{c}^{n}}-\mathrm{TZ}\right.$ or $\left.t_{x} \leq 5\right)$

The assigned states are maintained as the minimum green continuously, or until the lower bound of a transition zone is reached, regardless of the order or the combination of the signal groups.

B. $t_{x}>\frac{\hat{\phi}_{x}^{n}}{\zeta_{c}^{n}}-\mathrm{TZ}$, and $t_{x}>5$, and $t_{x}<\frac{\hat{\phi}_{x}^{n}}{\zeta_{c}^{n}}+\mathrm{TZ}$

If signal group $x$ is the preceding signal group in each pair, then the green time for signal group $x$ can be terminated to begin the green time for the following signal group in each pair, according to the pressure on each group in the given period. However, if signal group $x$ is the following signal group in each pair, then the current states of the signal controller (in which the green time is assigned in signal group $x$ ) and of the other paired signal group can be changed to terminate the current cycle, according to the pressure on the last signal groups in the current cycle and the first signal groups in the first clique for the future cycle. If the current state of traffic conditions satisfies Equations (44) and (45), then the current state of signal group $x$ (which is one of the first signal groups in the clique) is maintained. Otherwise, the inter-green time is assigned to signal group $x$. If the traffic conditions satisfy Equations (47a) and (47b), in which signal group $x$ is in the last signal group, then the right-of-way is continuously assigned to the last group in the current cycle. Otherwise, the current state of the signal controller is the inter-green time.

C. $t_{x} \geq \frac{\hat{\phi}_{x}^{n}}{\zeta_{c}^{n}}+\mathrm{TZ}$ 
The green time for signal group $x$ should be terminated with the inter-green time. Then, the green time is assigned to the following signal group in each pair, or to the first signal groups in the next cycle.

The current state of the signal controller can be changed within a transition zone according to the aforementioned rules, according to the pressure on each signal group, or on the basis of the cycle structure, because it is optimized in the global-optimization procedure. This developed group-based adaptive traffic-signal control logic can deal with both the global tendencies and the transient fluctuations in traffic flows by integrating the global-optimization method and the local adjustment of green time.

\subsection{Programs for the local reactive-control policy}

A local reactive-control policy is implemented by VBA to deal with the temporal variations in traffic conditions in the VISSIM simulation program. The rules developed for each group in each pair (or in each clique) are used to adjust the green time within the transition zones according to the traffic pressure. The combinations of the compared pressures are based on the values of the successor functions, as defined by the cycle structures and derived from the global-optimization procedure. In addition, the signal time zone is automatically set as a pivot of several seconds before and after the end time of the corresponding signal groups, as defined in the global-optimization method. The local adjustment of the green time operates within the defined zone. The aforementioned rules for each group, as described in Lee et al. (2017) and the previous sub-section, are simplified in the following figure.

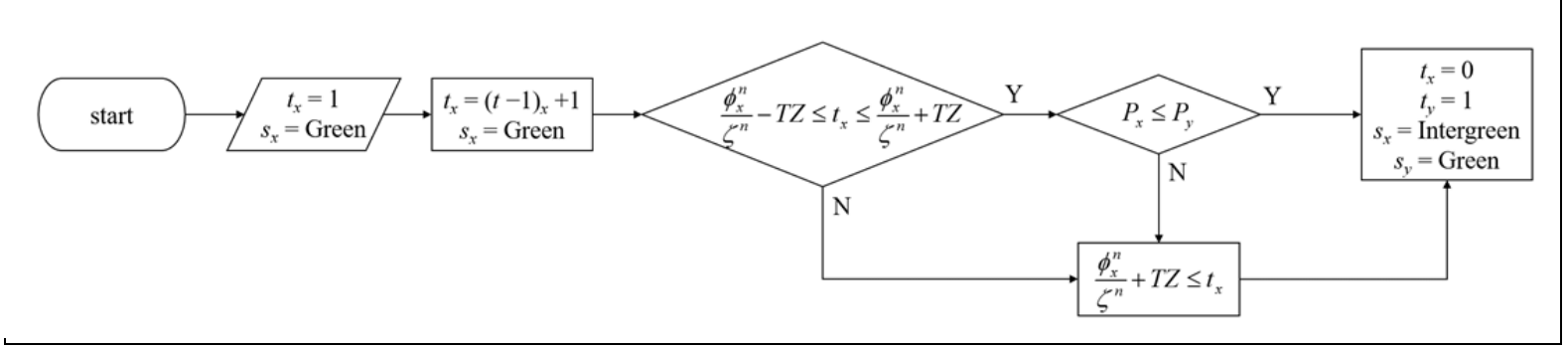

(a) In-pair

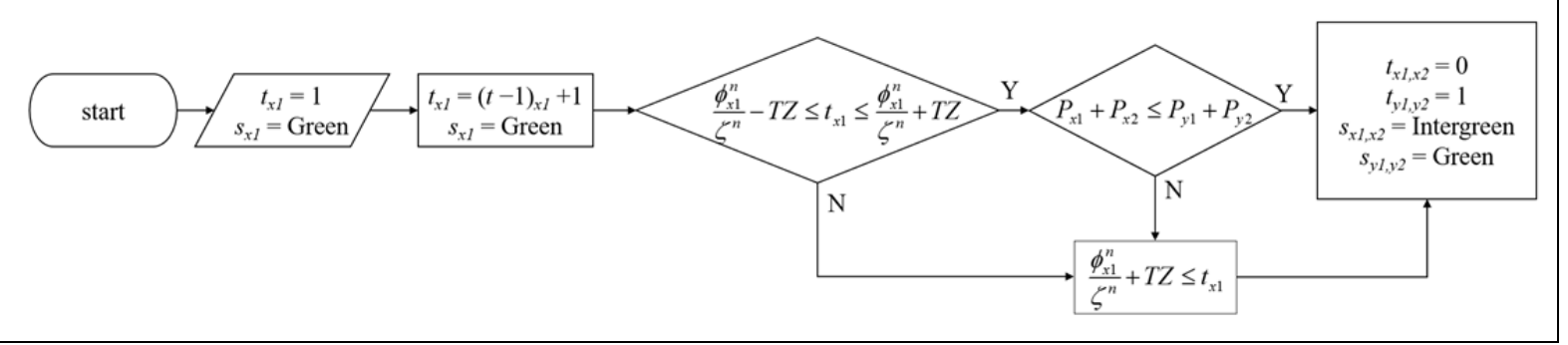

(b) Inter-clique

Figure 3. Simplified flowcharts for the local reactive-control policy

The current signal group $x$ follows the rules described in Figure 3(a) if group $x$ is the first signal group in each pair, as defined by successor functions. The initial setting of the signal controller (in which the green time is assigned to group $x$ ) is that the local clock of signal group $x$ starts running, and the current state of group $x$ is green time. Then, the pressure on group $x$ is compared with the pressure on the other group in the same pair if the local clock is within a transition zone. Based on the result of this comparison, the signal controller decides whether to extend a single second or to terminate the green time immediately for signal group $x$. After a transition zone, signal group $x$ enters 
the inter-green time immediately, and the local clock of group $x$ changes to 0 , whereas the green signal is assigned to group $y$ and the local clock of that group starts running.

If signal group $x l$ belongs to the last signal groups for each pair and each clique, then the current signal group $x 1$ follows the flowcharts as shown in Figure 3(b), with the compatible signal group $x 2$ in a clique. The max-pressure control policy starts working to adjust the current green time when the signal groups $x 1$ and $x 2$ reach the lower bounds of a transition zone. In addition, the current state of the signal groups is in the inter-green time if the pressure on the couple of signal groups $y 1$ and $y 2$ is larger than that on signal groups $x 1$ and $x 2$ during the transition zone. After the upper bound of a transition zone is passed, the current green time is immediately terminated, and the green time is assigned to the next signal groups in the consecutive clique.

\section{NUMERICAL EXAMPLES}

\subsection{Example settings}

The use of a simulation platform is one possible alternative for evaluating and verifying the performance of the newly developed traffic-control algorithms. The main advantages of a simulation platform approach are that it helps to generate identical inputs for the evaluation of different algorithms, avoids the high installation and operational costs involved in a real-traffic system, and is free from the safety concerns over conflicting traffic movements during the development stage. The traffic, signal, and geometrical information for this network is collected and implemented using the VISSIM microscopic simulation platform.

A typical directional three-lane, four-arm approach to an isolated intersection was constructed (with VISSIM computer software) to simulate the performance of the developed optimization method. A four-phase signalized intersection was created with control algorithms based on the component object model $(\mathrm{COM})$ interface. A full set of $2 \mathrm{~m} \times 2 \mathrm{~m}$ loop detectors was installed to record the impulse memory and time-occupancy-rate data. The upstream detectors were set back at $200 \mathrm{~m}$ from the stop-line. Left turns on red (LTOR) were prohibited, in accordance with the driving rule for lefthand traffic. The geometric layout of the simulated intersection is presented in Figure 4. The basic stage structure for the fixed-signal control was east-bound, west-bound, south-bound, and northbound. Each stage had three traffic streams: left-turn, right-turn, and passing-through traffic.

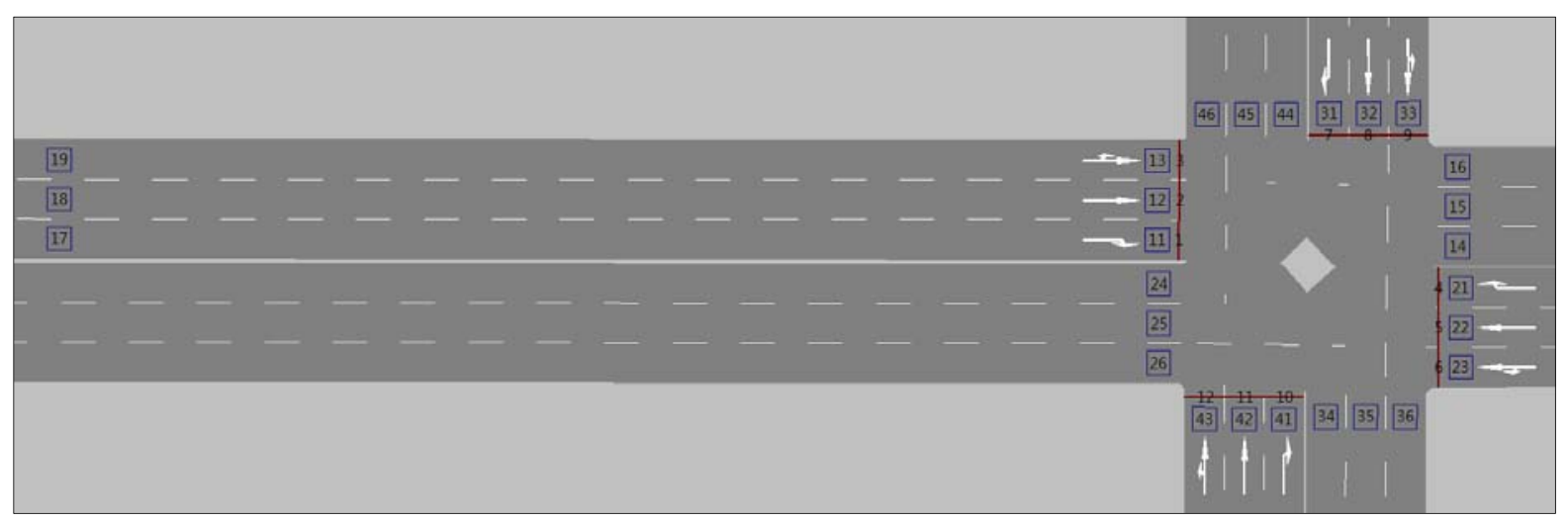

Figure 4. Geometric layout of the simulation junction in VISSIM 


\subsection{The appropriate transition zone}

To estimate the most appropriate transition zone, the simulation period was set as 18,000 seconds, the simulation resolution was 1 second, and the first and last 2,000 seconds were the warming-up and cooling-down periods. The turning proportions were randomly distributed among all of the movements for asymmetric traffic conditions. Seven levels of traffic volume (degrees of saturation), i.e., 700 (0.8), 750 (0.86), 800 (0.92), 850 (0.97), 900 (1.03), 950 (1.09), and 1000 (1.14) $\mathrm{vph}$, were set on all of the approaches in each 2,000-second interval to calculate the most suitable transition zone.

To validate the effectiveness of the proposed methodology in determining the suitable transition zone, five models were developed to minimize the control delay. These models were the output of the simulation program. Delay was defined as the difference between the free-flow travel time and actual travel time in VISSIM. Vehicle delay, measured in seconds, was used as the performance index. The first model was used as a control. This first model was an optimized fixedtime signal schedule based on the average traffic intensity and using a group-based approach that is commonly used in many countries to establish fixed-time signal plans. The transition zone for local adjustments was applied differently on the four other models, from zero to four seconds. The results in terms of vehicle delay and the stop-delay from the six models of asymmetric traffic conditions are given in Tables 5 and 6.

Table 5. The results on vehicle-delay (seconds) for the six models

\begin{tabular}{c|rrrrrr}
\hline $\begin{array}{c}\text { Simulation } \\
\text { time }\end{array}$ & \multicolumn{1}{|c}{ Fixed } & \multicolumn{5}{c}{ Proposed model with different transition zones } \\
\hline $0-2,000$ & 21.26 & 27.60 & 29.56 & 28.41 & 36.18 & 33.29 \\
$2,000-4,000$ & 22.16 & 33.88 & 28.65 & 31.45 & 27.96 & 32.77 \\
$4,000-6,000$ & 22.36 & 31.95 & 29.81 & 31.53 & 28.67 & 28.77 \\
$6,000-8,000$ & 24.24 & 34.27 & 29.64 & 33.52 & 34.46 & 36.22 \\
$8,000-10,000$ & 27.51 & 40.61 & 38.85 & 37.82 & 36.92 & 36.42 \\
$10,000-12,000$ & 72.72 & 48.96 & 41.22 & 42.13 & 47.63 & 41.10 \\
$12,000-14,000$ & 138.37 & 46.06 & 47.56 & 46.78 & 44.09 & 44.79 \\
$14,000-16,000$ & 318.27 & 55.52 & 70.70 & 64.23 & 56.13 & 62.83 \\
$16,000-18,000$ & 702.69 & 115.25 & 109.17 & 108.32 & 86.41 & 112.41 \\
TOTAL & $1,349.59$ & 434.12 & 425.16 & 424.19 & 398.45 & 428.61 \\
\hline
\end{tabular}

When the transition zone was set as three seconds before and after the end time of a signal group, the proposed algorithm performed well in all periods of analysis in minimizing the vehicle delay, as shown in Table 5. The total delay for the fixed-signal plan as established by the group-based approach (which considered the average traffic intensity for the whole analysis period) was 1,349.59 seconds, which was the poorest performance of all the models. All of the proposed models with ranges for their transition zones performed better than the fixed-signal plan. When a transition zone was 2 or 3 seconds either side of the end time for a stage, the performance of these models, at 398.45 seconds and 424.19 seconds of vehicle delay, respectively, was better than that of the fixed-control system. The proposed model with a 3 second transition zone did not give the best result for the total level of demand. The models with 2 and 3 second transition zones showed the lowest levels of total delay under both lower demand and over-saturated conditions.

Table 6. The results on stop-delay (seconds) for the six models

\begin{tabular}{l|ll} 
Simulation & Fixed & Proposed model with different transition zones
\end{tabular}




\begin{tabular}{|c|c|c|c|c|c|c|}
\hline time & & $0 \mathrm{sec}$. & $1 \mathrm{sec}$. & $2 \mathrm{sec}$. & $3 \mathrm{sec}$. & $4 \mathrm{sec}$. \\
\hline $0-2,000$ & 15.91 & 22.41 & 24.28 & 23.21 & 30.78 & 28.03 \\
\hline $2,000-4,000$ & 16.44 & 28.04 & 22.93 & 25.69 & 22.40 & 26.79 \\
\hline $4,000-6,000$ & 16.48 & 25.67 & 23.66 & 25.53 & 22.66 & 22.76 \\
\hline $6,000-8,000$ & 17.61 & 27.88 & 23.28 & 27.04 & 27.68 & 29.46 \\
\hline $8,000-10,000$ & 20.25 & 33.25 & 31.75 & 30.33 & 29.82 & 29.16 \\
\hline $10,000-12,000$ & 56.77 & 39.55 & 33.38 & 33.98 & 38.96 & 32.98 \\
\hline $12,000-14,000$ & 109.21 & 37.77 & 39.25 & 37.97 & 35.73 & 35.97 \\
\hline $14,000-16,000$ & 242.11 & 45.20 & 57.21 & 52.36 & 45.09 & 50.24 \\
\hline $16,000-18,000$ & 521.77 & 91.78 & 86.02 & 85.87 & 69.38 & 87.00 \\
\hline TOTAL & $1,016.57$ & 351.53 & 341.77 & 341.99 & 322.50 & 342.39 \\
\hline
\end{tabular}

From the stop-delay results shown in Table 6, the performance of the proposed models was distinctly improved compared to the fixed-signal plan. When the transition zone was set as 3 seconds before and after the end time of a stage, the proposed algorithm performed very well in all periods of analysis for asymmetric traffic conditions. The stop delay of the fixed-signal plan was $1,016.57$ seconds, which was the poorest performance. All of the models with a range of transition zones performed better than the fixed-signal plan. A transition zone of 3 seconds around the end time of a signal group resulted in much better performance than the fixed-signal control, with a total delay of only 322.5 seconds. The model with a 3 second transition zone attained the best overall performance, although it did not produce the best results for all levels of demand. The vehicle-delay and stop-delay results indicated that the use of a 3 second transition zone achieved the best performance.

\subsection{Scenario design for the simulation}

To validate the effectiveness of the proposed methodology, five models were developed to minimize the total delay, which was the measured output of the simulation program for the various simulations in VISSIM. The delay was defined as the difference between the free-flow travel time and the actual travel time in VISSIM. Total delay, expressed in (veh-sec), was used as the performance index. The first and second models were used as controls. The first model was an optimized fixedtime signal schedule, based on the average traffic intensity and using the group-based approach. This type of model is commonly used in many countries to establish fixed-time signal plans. The second model was an actuated signal-control logic model developed from the VISSIM software and based on data on headway and occupancy. The third model was the proposed global proactive-optimization method, and the fourth model was the local reactive-control policy. The last model was an integrated form of the global and local methods, which was used to consider both long-term and short-term fluctuations in traffic volume.

A total of 240 scenarios were simulated to test the abovementioned models. To verify the effectiveness and robustness of the proposed methods, the 240 scenarios were based on different traffic volumes and turning proportions for each approach. The proposed methods were able to explain not only the large changes in numerical differentiation under high levels of congestion, but also accounted for the tiny fluctuations observed under low levels of congestion. In addition, the different turning proportions on each approach indicated the robustness of the proposed methods under both symmetric and extremely asymmetric traffic conditions. Six levels of traffic volume (degrees of saturation), i.e., 175 (0.2), 350 (0.4), 525 (0.6), 700 (0.8), 875 (1.0), and 1,050 (1.2) vph, were set on all of the approaches for each case, and these levels were systematically combined according to the rules shown in the following table. 
Table 7. The combinations of traffic volume conditions

\begin{tabular}{cclc}
\hline No. & Condition & \multicolumn{1}{c}{ Description } & No. of cases \\
\hline 1 & Symmetric & same volumes over all approaches & 6 \\
2 & One-link asymmetric & differing volume for one approach & 30 \\
3 & Two-link asymmetric & differing volumes for a pair of approaches & 15 \\
4 & Fully asymmetric & differing volumes over all approaches & 9 \\
\hline
\end{tabular}

Table 7 shows that the first combination of traffic volumes was based on symmetric traffic assigned over all approaches, and there were six combinations for this condition. The second condition involved a certain level of traffic volume being assigned to one approach, with a different level of traffic volume on all of the other approaches. The number of combinations of traffic volume for this condition totaled 30 cases. In the third condition, a principal arterial route coexisted with a minor arterial route, and the proposed methods verified 15 cases for this combination. The last set of traffic conditions involved fully asymmetric traffic volumes over all approaches, and nine combinations were found to meet this description.

In terms of the combinations of turning proportions for each approach, there were four cases involving differing levels of symmetric or asymmetric turning proportions. The most extremely biased cases involved $70 \%$ of the traffic flows accessing the approach and assigned to one traffic movement. In other cases, specific traffic movements to access an approach were assigned to only $10 \%$ of the total traffic stream. A more detailed explanation of these turning proportions is outlined in the following table.

Table 8. The combinations of turning proportions

\begin{tabular}{c|ccc|ccc|cccc|ccc}
\hline \multirow{2}{*}{ No. } & \multicolumn{3}{|c|}{ Approach 1 } & \multicolumn{3}{|c|}{ Approach 2 } & \multicolumn{3}{c}{ Approach 3 } & \multicolumn{3}{c}{ Approach 4 } \\
& $\mathrm{T}$ & $\mathrm{L}$ & $\mathrm{R}$ & $\mathrm{T}$ & $\mathrm{L}$ & $\mathrm{R}$ & $\mathrm{T}$ & $\mathrm{L}$ & $\mathrm{R}$ & $\mathrm{T}$ & $\mathrm{L}$ & $\mathrm{R}$ \\
\hline 1 & 0.33 & 0.33 & 0.33 & 0.33 & 0.33 & 0.33 & 0.33 & 0.33 & 0.33 & 0.33 & 0.33 & 0.33 \\
2 & 0.50 & 0.30 & 0.20 & 0.20 & 0.50 & 0.30 & 0.50 & 0.30 & 0.20 & 0.30 & 0.50 & 0.20 \\
3 & 0.70 & 0.10 & 0.20 & 0.33 & 0.33 & 0.33 & 0.20 & 0.20 & 0.60 & 0.30 & 0.60 & 0.10 \\
4 & 0.40 & 0.10 & 0.50 & 0.70 & 0.10 & 0.20 & 0.60 & 0.10 & 0.30 & 0.60 & 0.30 & 0.10 \\
\hline
\end{tabular}

Table 8 shows that the first case was based on symmetric conditions, with one-third of the vehicles being assigned to each turning movement. In the second case, $50 \%$ of the traffic volumes were assigned to the passing-through, left-turning, passing-through, and right-turning movements on approaches $1,2,3$, and 4 , respectively. In the third case, $70 \%$ of the traffic flow was assigned to passing-through movements on approach 1 , and the vehicles were evenly distributed among the right and left turning movements. On approaches 3 and 4, 60\% of the vehicles were assigned to right-turn and left-turn movements, respectively. In the fourth case, a small proportion $(10 \%)$ of the vehicle flow was assigned to the left-turning movements over all approaches, and differing levels of flow were assigned to the other movements on each approach.

These combinations of traffic flows and turning proportions generated 240 different cases $(60 \times 4$ cases) with which to validate the robustness and effectiveness of the proposed methods. The simulation period was 2,400 seconds, the simulation resolution was 1 second, and the first and last 300 seconds were the warming-up and cooling-down periods for each case. 


\subsection{Numerical results}

The performance of the proposed methods (i.e., the global proactive-optimization method, the local reactive-control policy, and the integrated adaptive traffic-signal control) was compared with the performance of the widely used signal control, fixed-timing control, and actuated-control methods. The measures of effectiveness (MOEs) for these models (on the isolated signalized junction provided by VISSIM) were the number of stops, the stopped delay, the vehicle delay, and the number of vehicles handled by the target intersection. VISSIM defined the number of stops as the average number of vehicle stops per vehicle, omitting stops at public transport (PT) stops or in parking lots. Stopped delay was defined as the average stopped delay per vehicle in seconds, omitting stops at PT stops or in parking lots. Vehicle delay was defined as the average delay for all vehicles. In using measured delay as one of the MOEs, VISSIM obtained the delay for a vehicle by subtracting its theoretical (ideal) travel time from its actual travel time. The theoretical travel time was the travel time that could be achieved if there were no other vehicles, and/or no signal controls, or any other reasons for stops. Reduced-speed areas were taken into account. The actual travel time did not include any passenger service times for PT vehicles at stops or any parking time in real parking lots. Any delays due to braking before a PT stop and/or subsequent acceleration after a PT stop were considered as part of the total delay.

The first model was an optimized fixed-time-signal schedule based on the average traffic intensity and using a group-based approach, as is commonly used in many countries to establish fixed-time-signal plans. This first model was the reference model, which was patterned on the groupbased approach to the fixed-signal control plan, and was described as the "Fixed" model. A cycle length of 60 seconds was provided with a green signal duration of 10 seconds for each phase and an inter-green time of 5 seconds.

The second model, which was called the "Actuated" model, represented an actuated signalcontrol logic. This kind of control logic is one of the most widely used non-fixed-signal-control methods derived from VISSIM software. The model was based on headway and occupancy data that determined whether to extend the green time by three seconds, or to terminate the current green time immediately. These first two models were used as controls.

The proposed proactive global-optimization scheme was considered as the third model, which was called the "Global" model. This scheme is described in Section 4. The fourth model was the local reactive-control policy, which is described in Section 5, and is called the "Local" model. The fifth model was the integrated group-based adaptive traffic-signal control, which is the most complicated form of control logic. This model was based on both the global-optimization method and the local control policy, and was described as the "Integrated" model. The time windows for the proactiveoptimization method consist of the next, current, and previous cycles. The start and duration of the green time and the cycle time for the next cycle are determined for the current cycle using the mathematical predictive and optimization frameworks applied to each cycle. The prediction of future patterns is based on the detector data collected from the previous cycle. Thus, we guarantee the current cycle for the computational time of the non-linear and linear programs. The computational times, around 10 to 15 seconds, were sufficiently short for a general cycle length to bear.

Statistical information on the simulation results from these models (i.e., the fixed-control, actuated control, global optimization, local control policy, and integrated adaptive control logic) are summarized in the following table. 
Table 9. Statistical information on the simulation results of the models

\begin{tabular}{|c|c|c|c|c|c|c|}
\hline MOE & Stat. & Fixed & Actuated & Global & Local & Integrated \\
\hline StopDelay & Total & 70,993 & 55,171 & 24,850 & 31,358 & 22,396 \\
\hline (seconds) & Avg. SD & $314.1 \quad 255.0$ & 244.1202 .0 & 110.0136 .7 & $138.8 \quad 185.0$ & $99.1 \quad 135.6$ \\
\hline Stops & Total & 10,320 & 6,123 & 3,091 & 4,937 & 2,492 \\
\hline (stops) & Avg. SD & $\begin{array}{ll}45.7 \quad 39.6 \\
\end{array}$ & $27.1 \quad 26.1$ & 13.7 & $21.8 \quad 32.9$ & $\begin{array}{ll}11.0 & 19.0 \\
\end{array}$ \\
\hline VehDelay & Total & 93,352 & 69,217 & 32,845 & 43,521 & 28,939 \\
\hline (seconds) & Avg. SD & $413.1 \quad 332.5$ & $\begin{array}{ll}306.3 & 255.7\end{array}$ & $145.3 \quad 178.9$ & $192.6 \quad 253.4$ & $\begin{array}{ll}128.0 & 173.7\end{array}$ \\
\hline Vehs & Total & 165,282 & 165,360 & 174,968 & 177,503 & 173,972 \\
\hline (vehicles) & Avg. SD & $731.3 \quad 205.5$ & $\begin{array}{lll}731.7 & 218.8\end{array}$ & $774.2 \quad 246.0$ & $\begin{array}{ll}785.4 & 222.7\end{array}$ & $\begin{array}{ll}769.8 & 257.4\end{array}$ \\
\hline
\end{tabular}

Table 9 shows that the proposed three models performed better than the fixed-signal plan and the actuated control in terms of the total stopped delay, number of stops, vehicle delay, and numbers of vehicles served. According to the representative MOE, the performance of the fixed-signal plan (as established by the group-based approach and based on the average traffic intensity for all cases of analysis) was 93,352 seconds, which was the poorest performance among all of the models. The performance of the actuated signal control was much better than that of the fixed-signal plan, with the total vehicle delay being 69,217 seconds. The total vehicle delay for the global-optimization method and the local control policy was 32,845 and 43,521 seconds, respectively, both of which were considerably lower than the delays for the fixed and actuated signal controls. The integrated adaptive traffic-signal control performed especially well under diverse traffic conditions. The total vehicle delay for the integrated model was 28,939 seconds, which was almost one-third of that of the fixedsignal control.

Figure 5 shows the time-varying cycle structure obtained from the integrated adaptive traffic control systems, which comprise the global proactive optimization method and the local reactive control policy in case 1 of the combinations of turning proportions. The y-axis shows the signal groups (SG) from 1 to 12 and the $\mathrm{x}$-axis describes seconds of time.

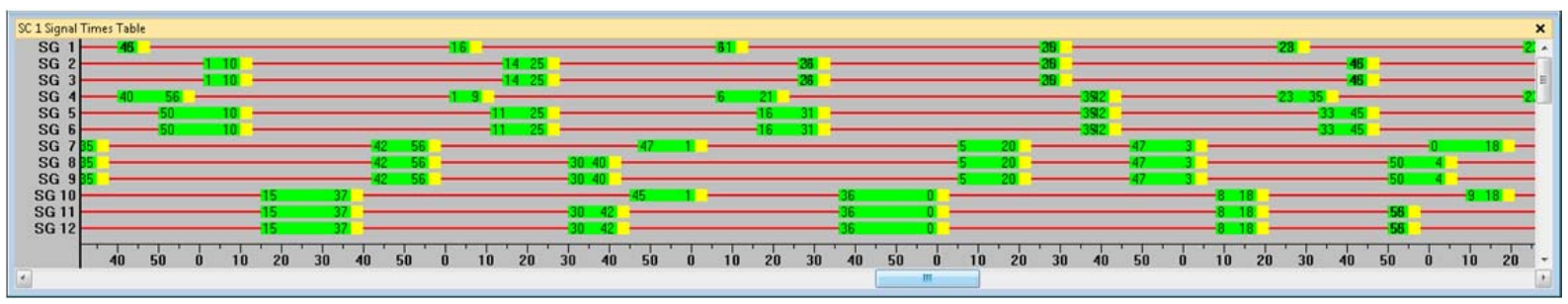

Figure 5. Example of the time-varying cycle structure from the integrated method

In Figure 5, the first signal group is fixed for each cycle, and the rest of the signal groups are rearranged to minimize the total delay with the cycle-varying successor functions in every cycle based on the real-time traffic information. The cycle structure, including the duration of green time, signal sequence, and cycle time, is determined by the global proactive optimization method, whereas the actual green time is adjusted by the local reactive control policy within a transition zone.

The data on the total stopped delay and the numbers of stops showed that the performance of the proposed methods was much better than that of the existing methods, and the integrated adaptive traffic-signal-control logic performed well in diverse traffic conditions. The numbers of vehicles served by the target intersections among the compared five methods were similar, with only a $7 \%$ range of difference. The vehicle delay for the existing and the proposed methods (with respect to the 
combinations of turning flows on each approach as shown in Table 8) are presented in the following table.

Table 10. The average vehicle delay (seconds) for the models, by cases

\begin{tabular}{crrrrr}
\hline Case & \multicolumn{1}{c}{ Fixed } & \multicolumn{1}{c}{ Actuated } & \multicolumn{1}{c}{ Global } & \multicolumn{1}{c}{ Local } & \multicolumn{1}{c}{ Integrated } \\
\hline 1 & 109.30 & 37.64 & 46.41 & 77.46 & $\mathbf{3 5 . 8 0}$ \\
2 & 549.38 & 469.22 & 104.52 & 115.13 & $\mathbf{5 7 . 7 8}$ \\
3 & 609.27 & 450.07 & $\mathbf{3 3 0 . 6 2}$ & 369.14 & 337.39 \\
4 & 360.26 & 217.79 & 73.81 & 172.71 & $\mathbf{5 5 . 6 9}$ \\
\hline
\end{tabular}

Cases 1, 2, 3, and 4 imply symmetric, one-link asymmetric, two-link symmetric, and fully asymmetric traffic conditions, respectively, as described in Tables 7 and 8 . Table 10 shows that the three proposed methods performed impressively better than the existing control methods, except in case 1 , which was based on symmetric traffic conditions. In case 1, the vehicle delays for the actuated control (37.64 seconds) and those of the proposed methods where much better than the delay under the fixed-control plan (109.30 seconds). The global optimization and local control policy performed worse (46.41 and 77.46 seconds, respectively) than the actuated control. The integrated adaptivesignal control showed the lowest vehicle delay ( 35.80 seconds) under symmetric traffic conditions. In cases 2 and 4, the integrated model performed better than the other models, with delays of 57.78 and 55.69 seconds, respectively. The global proactive-optimization method showed the second-best vehicle-delay results, with 104.52 and 73.81 seconds in cases 2 and 4, respectively. The performance of the local reactive-control policy was the worst among the proposed methods, with delays of 115.13 and 172.71 seconds, respectively. Still, this was a much better performance than that of the existing methods, with the fixed control having 549.38 and 360.26 seconds delay, and the actuated control having 469.22 and 217.79 seconds delay, respectively. Both the integrated model and the globaloptimization method performed well in case 2, with 337.39 and 330.62 seconds, respectively. The local reactive-control policy gave the third-best results, with 369.14 seconds delay. The performance of the existing methods was not impressive, with 609.27 and 450.07 seconds delay, respectively. The detailed tendencies of the vehicle delays in each case are examined in the following figure.

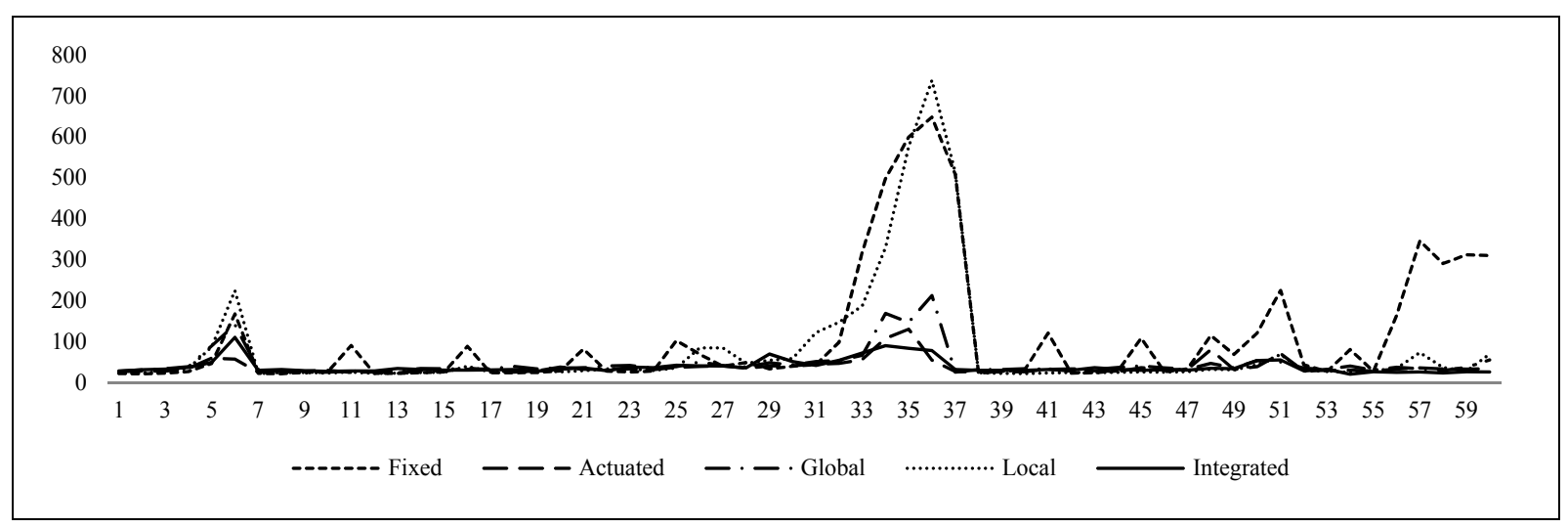

(a) Case 1 on the combination of turning proportions 


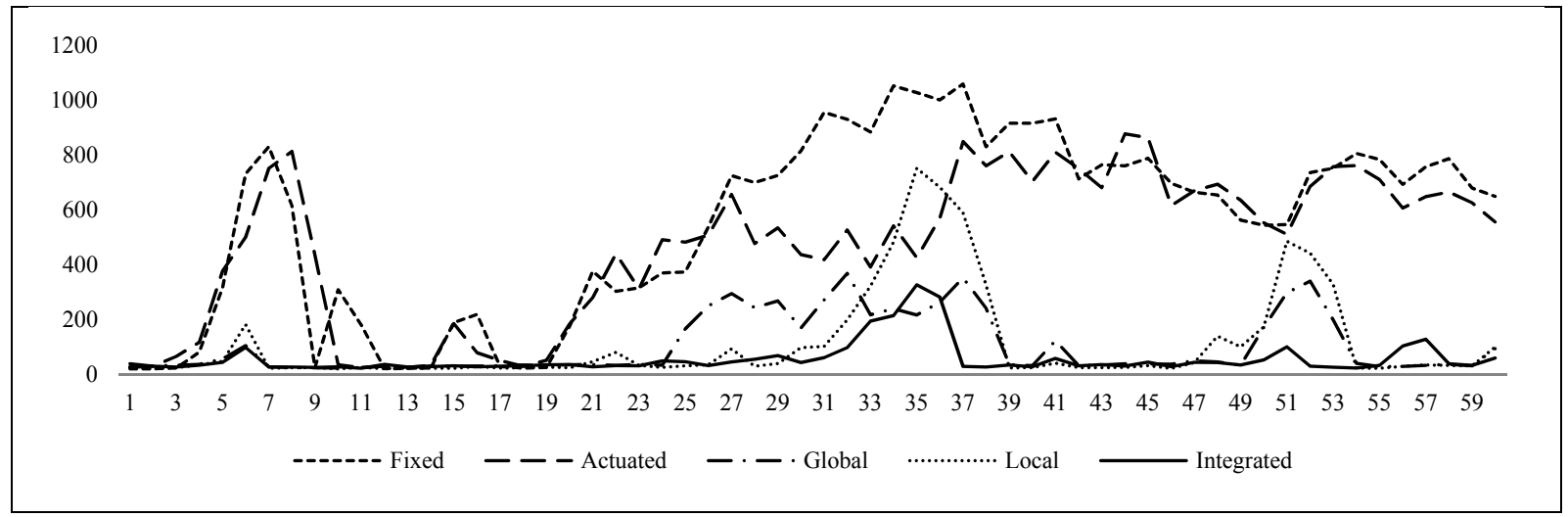

(b) Case 2 on the combination of turning proportions

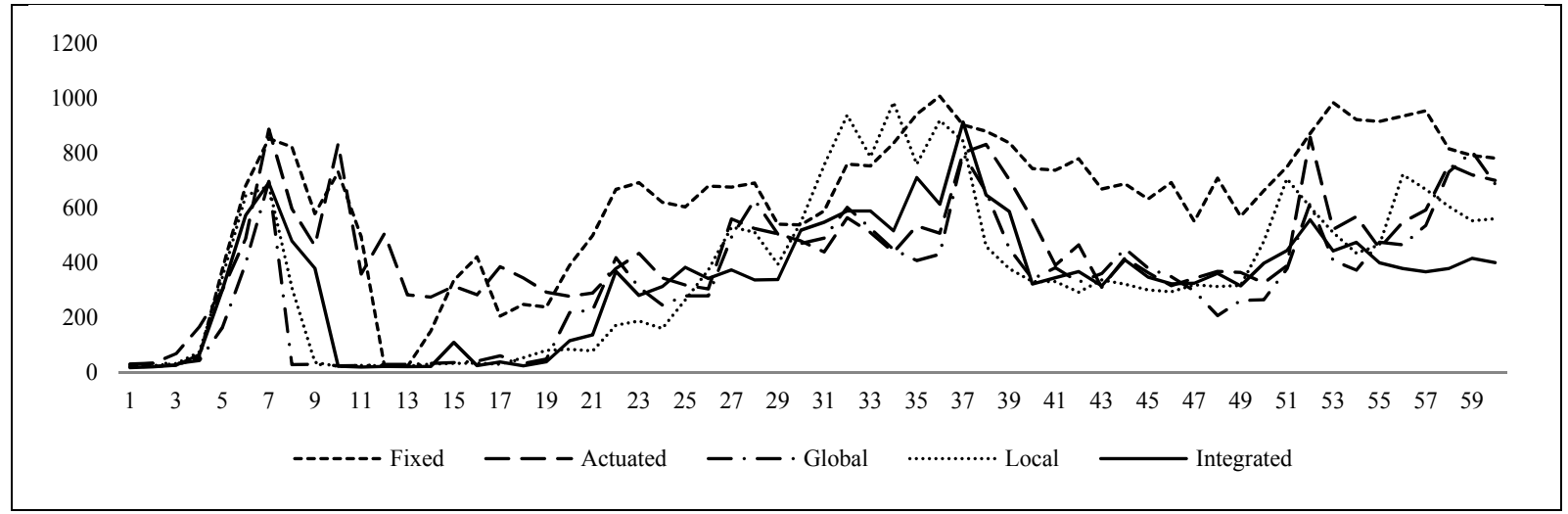

(c) Case 3 on the combination of turning proportions

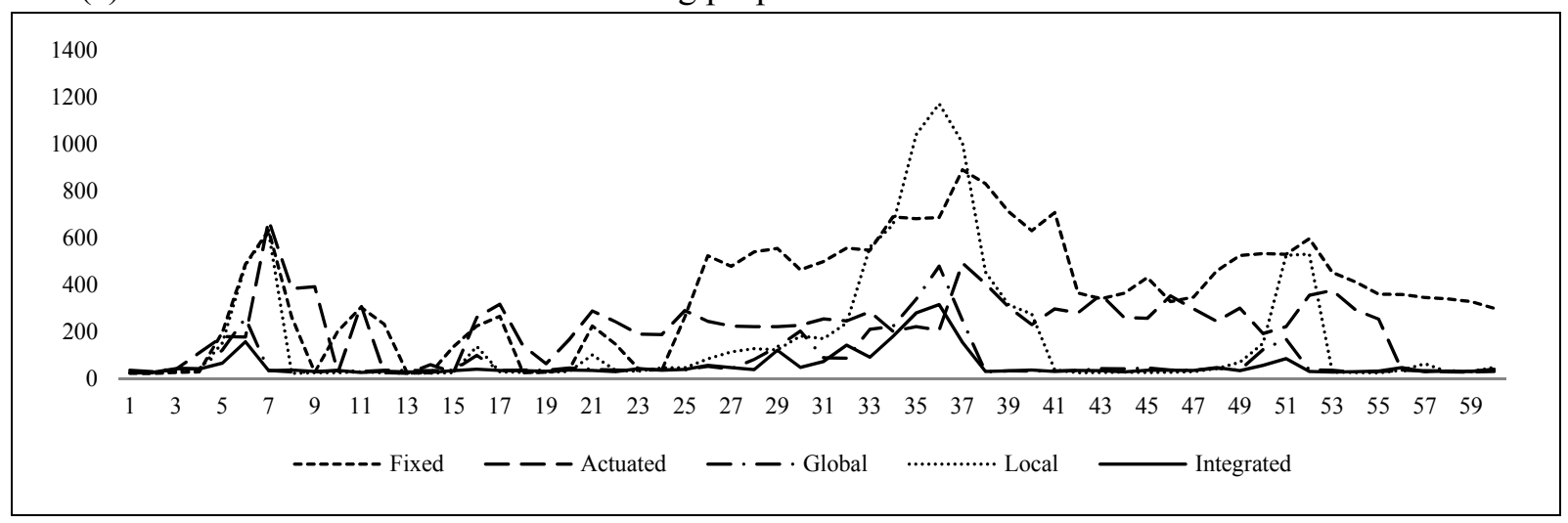

(d) Case 4 on the combination of turning proportions

Figure 6. The vehicle delays (seconds) from the compared methods, by case

In Figures 6(a) - 6(d), the y-axis shows the average vehicle delay (seconds) and the x-axis describes the 60 different traffic conditions, from symmetric to fully asymmetric patterns, given in Table 7.

Figure 6(a), indicates that the patterns for the control delay were similar for all models, with differing degrees of delay under symmetric traffic conditions in terms of the turning proportions. The local reactive-control policy performed similarly to the fixed-control plan when the cases with the most asymmetric traffic conditions on each approach (cases 31-39) were omitted. However, the actuated control, global proactive-control policy, and integrated model provided enhanced the performance over all of the cases, with only slight differences in the control delay.

Figure 6(b) and (d) shows that the performance of the proposed methods was a definite improvement over the existing methods, namely the fixed and actuated control systems. The proposed 
methods showed enhanced control over the delay under all conditions, even as the asymmetric conditions were intensified in terms of the traffic flows on each approach and the proportion of each type of traffic movement. In particular, the integrated adaptive traffic-control logic maintained a relatively even degree of congestion without deterioration in the levels of service under diverse traffic conditions, including steady free-flow conditions and extremely congested conditions, caused by heavy traffic volumes or asymmetric traffic proportions.

Figure 6(c) shows that the performance of the integrated model was not overwhelmingly better than that of the other models. However, all of the proposed methods definitely showed improved performance over the existing methods in all of the cases examined. In case 3, concerning the combinations of turning proportions, a high proportion of right-turning movements resulted in worse performance for the existing and proposed methods. In addition, none of the tested methods were capable of effectively handling the weaving movements between heavy passing-through and right-turning movements in the middle of the approach.

\section{CASE STUDY}

The proposed models were applied to high-resolution traffic data collected at the junction of Ladys Island Drive/Ribaut Road/State Road S-7-233 in South Carolina. The junction was actualized in the proposed simulation platform of the integrated adaptive traffic-signal-control logic. This highresolution traffic surveillance system was introduced in Muralidharan et al. (2016) and Coogan et al. (2017). The collected data set was used to calibrate the traffic conditions in the simulation platform. The geometric layout of the target junction and the location of the sensors for the high-resolution data set are described in Figure 7.

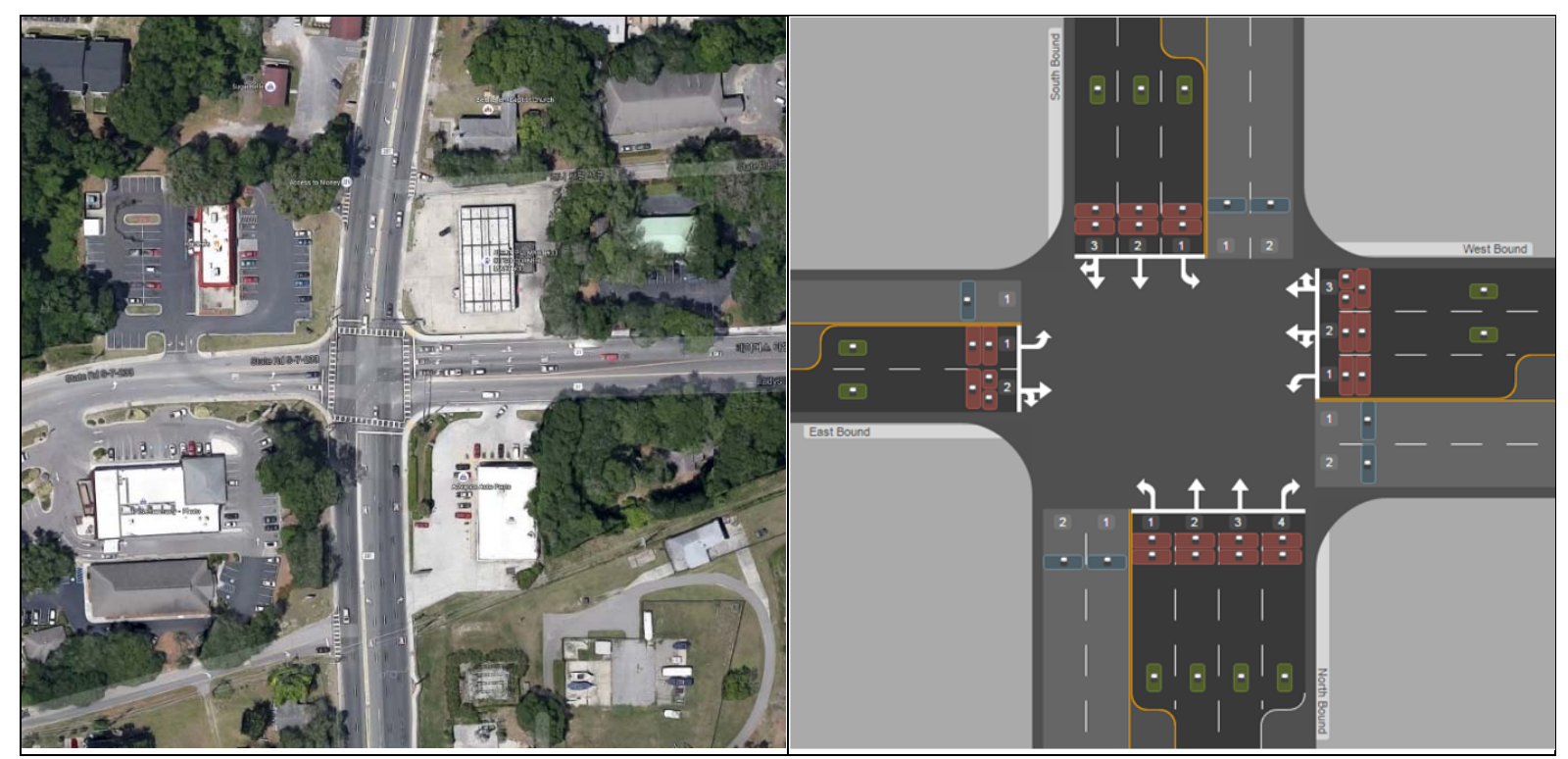

(a) The satellite image of the junction

(b) The locations of the sensors

Figure 7. The geometric conditions of the target intersection

The sensors were situated at all downstream (stop bar), exit, and partial upstream (advanced) lanes. A single sensor was used to collect the departure times and occupancy at an exit and an upstream lane. A pair of sensors was used to additionally collect speed data at all downstream lanes. The upstream sensors were set back at a distance of $225 \mathrm{ft}(68.58 \mathrm{~m}), 225 \mathrm{ft}(68.58 \mathrm{~m}), 330 \mathrm{ft}$ $(100.584 \mathrm{~m})$, and $250 \mathrm{ft}$ (76. 2m) for South Bound (SB), North Bound (NB), West Bound (WB), and East Bound (EB), respectively. There were two data sets of high-resolution traffic data, namely, 
PhaseData.txt and SensorData.txt. The sampling frequency was $16 \mathrm{~Hz}, 0.0625$ seconds. The analysis period was from 1430538921 to 1430623073 UTC (Coordinated Universal Time), or from 22:55:21 on May 1, 2015, to 22:17:53 on May 2, 2015 (Sat).

To calibrate the coefficients in the discriminant models and the parameters in the Kalman filter, the target intersection in the VISSIM simulation program was replicated because information about observed queue lengths was not provided. A four-phase signalized intersection with a cycle length of 60 seconds was created. The green signal duration for each phase was 10 seconds, with an inter-green time of 5 seconds. A full set of loop detectors was installed to record the impulse memory and time occupancy rate time. The simulation period was 36,000 seconds and the simulation resolution was 1 seconds. Furthermore, the last 20 cycles were the warming down period, and the impulse memories and occupancy rates collected during 580 cycles were used to calibrate the discriminant models and the Kalman filter. Then, the traffic volumes were randomly set to consider both a free flow traffic condition and a traffic congestion condition. It was further assumed that the turning proportions were evenly distributed among all of the movements. One-third of the vehicles were assigned to each turning movement for the model calibration. The layout of the target intersection is described below.

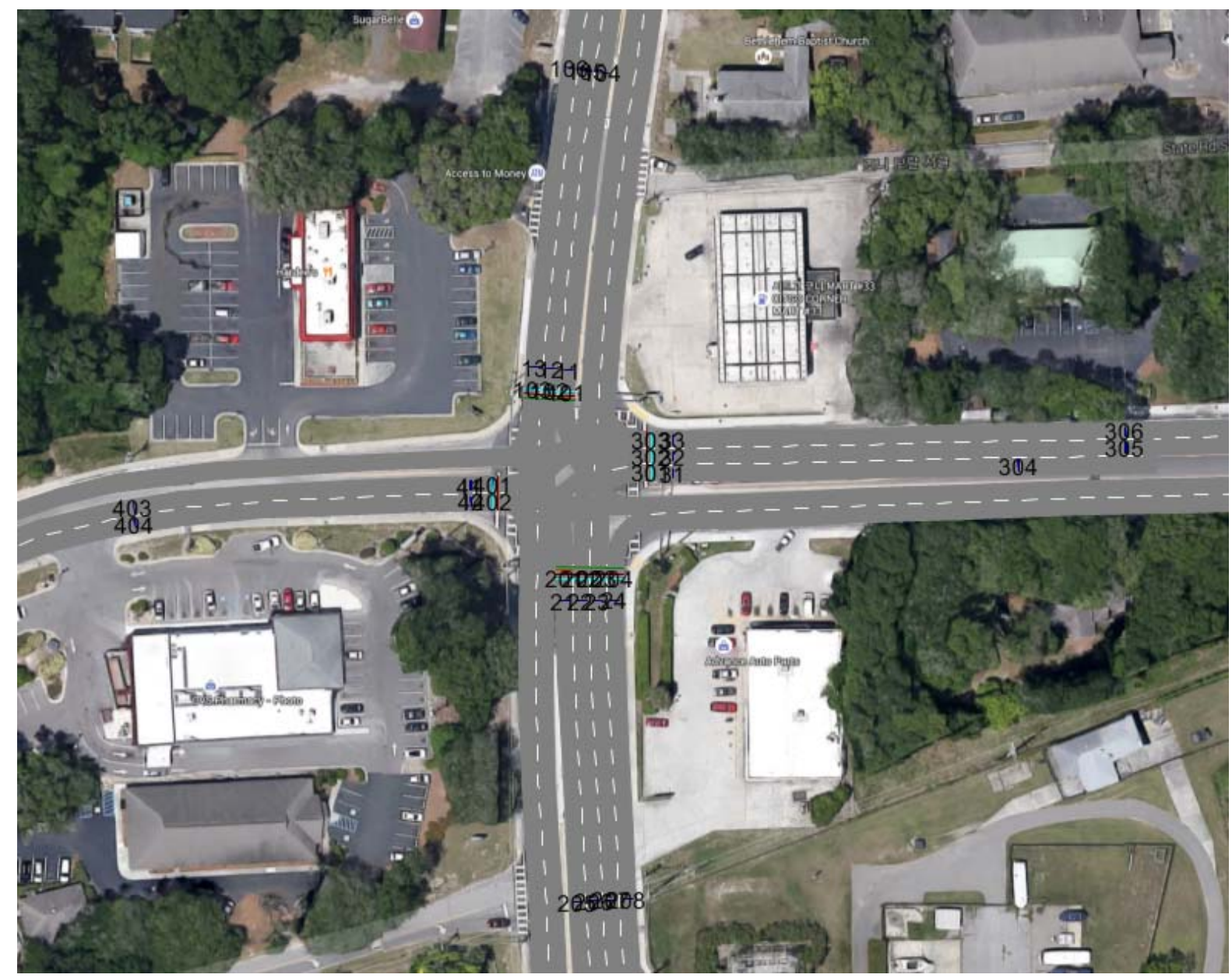

Figure 8. The layout of the target intersection in the VISSIM simulation environment

To verify the calibrated models using the proposed integrated adaptive traffic-signal-control logic, the pattern of the queued vehicles in the calibration sets was compared with the calculated speed based on the observed data from the upstream sensors. The calculated speed at the upstream sensors was assumed to be less than $5 \mathrm{~km} / \mathrm{h}$ when the vehicles queued over the upstream sensors, i.e., 
the number of estimated queued vehicles was greater than the capacity of queue lengths. The upstream speed was calculated using the estimated effective length of the sensors and the observed occupancy at the upstream sensors. A predicted blocking-back to the upstream sensors on each lane was compared with the observed blocking-back to the upstream sensors. Whether the calculated upstream speed was less than $5 \mathrm{~km} / \mathrm{h}$ was checked when the vehicles queued over the upstream sensors. The indicator for a predicted blocking-back to the upstream sensors was 1 second when vehicles queued over the upstream sensors, and 0 otherwise. The indicator for an observed blocking-back to the upstream sensors was 1 second when the upstream speed was below $5 \mathrm{~km} / \mathrm{h}$, and 0 otherwise. When both indicators were 1 or 0 at the same time, it was determined that the estimated queue lengths did not reflect the real traffic conditions well and the indicators for this inconsistency were set as 1 , and 0 otherwise. The results of the verification are presented in Table 11.

Table 11. The results of the verification

\begin{tabular}{c|rrr|rrrr|rrr|rr}
\hline Leg & \multicolumn{3}{|c|}{1} & \multicolumn{4}{|c|}{2} & \multicolumn{3}{|c}{3} & \multicolumn{2}{|c}{4} \\
Lane & 1 & 2 & 3 & 1 & 2 & 3 & 4 & 1 & 2 & 3 & 1 & 2 \\
\hline Total Time & 83958 & 83958 & 83958 & 83958 & 83958 & 83958 & 83958 & 83958 & 83958 & 83958 & 83958 & 83958 \\
Inconsistency & 1357 & 37 & 48 & 0 & 29 & 594 & 252 & 285 & 5807 & 1670 & 0 & 3 \\
Percentage & $1.62 \%$ & $0.04 \%$ & $0.06 \%$ & $0.00 \%$ & $0.03 \%$ & $0.71 \%$ & $0.30 \%$ & $0.34 \%$ & $6.92 \%$ & $1.99 \%$ & $0.00 \%$ & $0.00 \%$ \\
\hline
\end{tabular}

In Table 11, the total time is the analysis time. An inconsistency is the number of seconds when the indicator for the inconsistency is 1 . The percentage is the length over the total analysis time. A considerable proportion of the indicators for an observed blocking-back to the upstream sensors are similar to those for a predicted blocking-back to the upstream sensors, even if all of the indicators for an observed blocking-back to the upstream sensors do not exactly accord with those of the predicted blocking-back to the upstream sensors. Accordingly, it was determined that the traffic conditions in the calibration sets reflected the real world sufficiently well.

To validate the performance of the proposed group-based adaptive traffic-signal control, the validation sets were designed based on both the observed traffic conditions in the real world and the calibrated models in the proposed simulation platform. The traffic flows for each lane for each leg were extracted from the high-resolution surveillance system in the target junction. The detailed traffic flows with respect to the daily time periods are described below.

Table 12. The observed traffic flows from the high-resolution surveillance system

\begin{tabular}{rr|cccccccccccc}
\hline \multicolumn{2}{c|}{ Daily hour } & \multicolumn{3}{c}{ Leg 1} & \multicolumn{3}{c}{ Leg 2} & \multicolumn{3}{c}{ Leg 3} & \multicolumn{3}{c}{ Leg 4} \\
From & To & T & L & R & T & L & R & T & L & R & T & L & $\mathrm{R}$ \\
\hline $23: 00$ & $0: 00$ & 6 & 61 & 35 & 4 & 4 & 1 & 64 & 37 & 7 & 62 & 88 & 3 \\
$0: 00$ & $1: 00$ & 4 & 43 & 16 & 2 & 1 & 0 & 55 & 11 & 9 & 23 & 42 & 3 \\
$1: 00$ & $2: 00$ & 4 & 37 & 17 & 2 & 1 & 0 & 31 & 12 & 4 & 29 & 20 & 1 \\
$2: 00$ & $3: 00$ & 5 & 43 & 12 & 4 & 0 & 1 & 17 & 9 & 2 & 16 & 22 & 0 \\
$3: 00$ & $4: 00$ & 5 & 51 & 15 & 2 & 1 & 1 & 16 & 9 & 2 & 20 & 27 & 3 \\
$4: 00$ & $5: 00$ & 10 & 90 & 24 & 4 & 10 & 1 & 31 & 16 & 3 & 34 & 40 & 5 \\
$5: 00$ & $6: 00$ & 13 & 141 & 92 & 16 & 26 & 4 & 73 & 53 & 9 & 106 & 73 & 10 \\
$6: 00$ & $7: 00$ & 20 & 189 & 142 & 50 & 27 & 12 & 154 & 99 & 17 & 146 & 170 & 28 \\
$7: 00$ & $8: 00$ & 31 & 362 & 424 & 79 & 51 & 20 & 233 & 352 & 29 & 186 & 301 & 52 \\
$8: 00$ & $9: 00$ & 44 & 387 & 238 & 70 & 37 & 18 & 321 & 197 & 34 & 286 & 395 & 49 \\
$9: 00$ & $10: 00$ & 45 & 416 & 233 & 76 & 52 & 19 & 416 & 147 & 44 & 412 & 510 & 74 \\
$10: 00$ & $11: 00$ & 45 & 392 & 230 & 62 & 54 & 15 & 428 & 257 & 49 & 469 & 576 & 56
\end{tabular}




\begin{tabular}{cc|cccccccccccc}
$11: 00$ & $12: 00$ & 33 & 327 & 255 & 73 & 67 & 18 & 347 & 267 & 45 & 415 & 594 & 52 \\
$12: 00$ & $13: 00$ & 37 & 374 & 237 & 66 & 55 & 17 & 402 & 244 & 49 & 372 & 544 & 51 \\
$13: 00$ & $14: 00$ & 29 & 318 & 198 & 54 & 32 & 13 & 385 & 240 & 47 & 355 & 511 & 54 \\
$14: 00$ & $15: 00$ & 36 & 332 & 255 & 62 & 40 & 15 & 356 & 215 & 43 & 339 & 527 & 44 \\
$15: 00$ & $16: 00$ & 27 & 289 & 204 & 33 & 46 & 8 & 330 & 191 & 37 & 284 & 479 & 42 \\
$16: 00$ & $17: 00$ & 34 & 333 & 242 & 50 & 46 & 12 & 346 & 158 & 42 & 301 & 447 & 35 \\
$17: 00$ & $18: 00$ & 27 & 296 & 218 & 50 & 63 & 12 & 287 & 124 & 36 & 304 & 439 & 40 \\
$18: 00$ & $19: 00$ & 24 & 254 & 162 & 45 & 43 & 11 & 303 & 138 & 35 & 306 & 346 & 29 \\
$19: 00$ & $20: 00$ & 18 & 205 & 144 & 43 & 33 & 11 & 240 & 136 & 30 & 210 & 261 & 25 \\
$20: 00$ & $21: 00$ & 21 & 191 & 132 & 30 & 22 & 8 & 182 & 94 & 25 & 180 & 229 & 14 \\
$21: 00$ & $22: 00$ & 11 & 125 & 94 & 14 & 17 & 4 & 111 & 60 & 14 & 146 & 173 & 11 \\
\hline
\end{tabular}

In Table 12, the asymmetric traffic conditions are time-varying in the turning proportions and traffic volumes on each leg. The directions of the significant traffic flows are different in the morning peak-hours and evening peak-hours. In leg 1, a few vehicles go through over the entire period, while the ratio between the numbers of left and right turning vehicles differ slightly according to the daily time period. The traffic flows in leg 2 are relatively small, and most of the traffic flows in legs 3 and 4 are going through and left turning movements. These asymmetric and largely time-varying traffic conditions were considered to be suitable for the comparison between the existing models and the proposed model. The performance of the integrated adaptive traffic-signal control, which was finally recommended in the simulation environment, was compared with the performance of the existing fixed-signal plan and the actuated signal-control. The tendencies of the vehicle delays of each model are examined in the following figure.

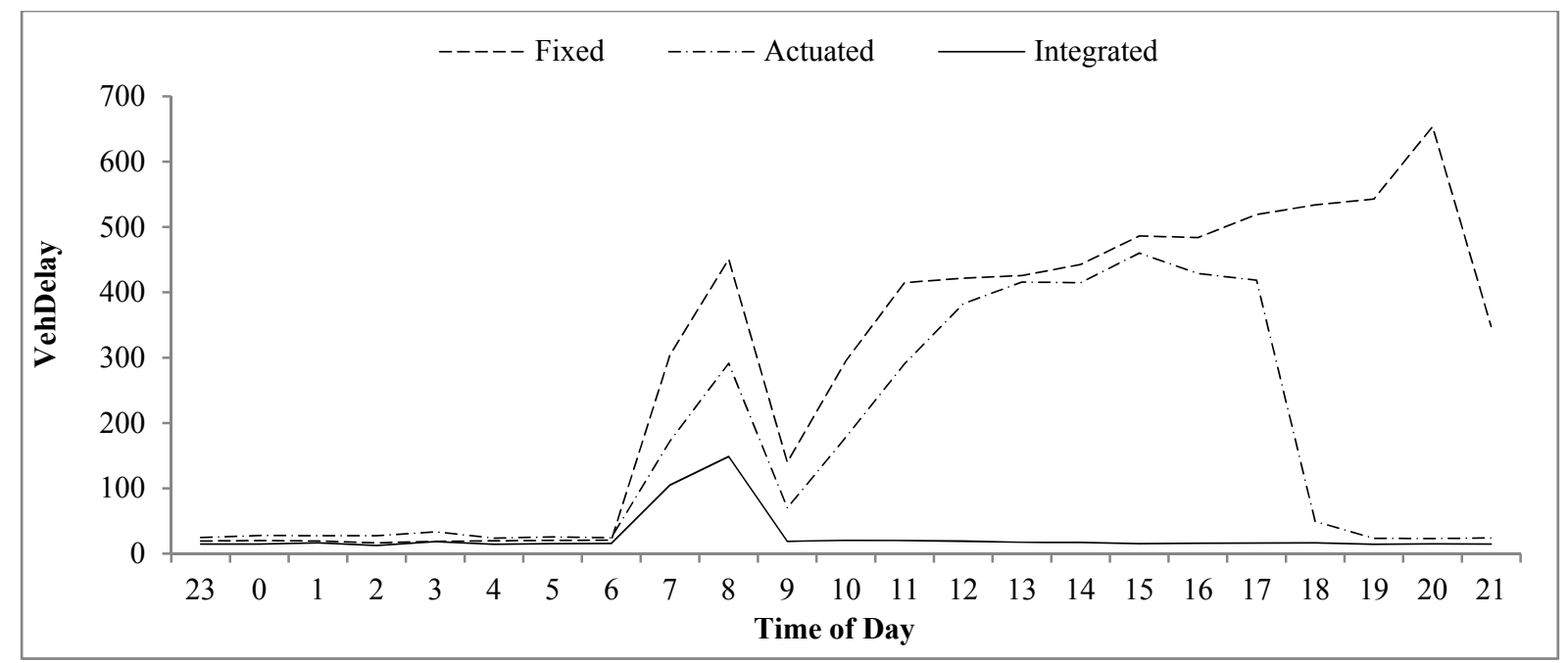

Figure 9. The vehicle delays (seconds) from the compared methods in the validation sets

In Figure 9, the performance of the proposed method is definitely enhanced over the existing methods over the whole day. Because the level of traffic flow at the junction was consistently high from morning to night, traffic congestion frequently occurred under the operation of the fixed signal schedule and the actuated control system. The integrated adaptive traffic-control logic attained a lower level of traffic congestion, without extreme deterioration in the levels of service, although the asymmetric conditions were intensified both in terms of the traffic flows on each approach and the proportions for each type of traffic movement according to the time of day. The large difference between the performance of the proposed model and the existing methods is likely to have been 
caused by the relatively high fluctuations in traffic flows on each approach and the turning proportions in each lane over the day.

To easily understand the effectiveness of the proposed integrated method, we approximately calculated the travel time saving benefit at the target intersection using average vehicle occupancy and values of travel time savings. The total vehicle delays for 24 hours of the fixed control and the proposed model are 12,819,334 and 1,056,148 seconds 'vehicles, respectively, and the saving time is $11,763,187$ seconds'vehicles. We used 1.67 persons per vehicles as average vehicle occupancy in Santos, A. et al. (2011) and USD 12.80 as recommended hourly values of travel time savings in Rogoff, P. and Ayala, P. (2014). Consequently, the approximate travel time saving benefit is USD 84,035 per day through the installation of the proposed method at the intersection and the proposed group-based ATCS evidently improved the performance of the target intersection to efficiently handle under- and oversaturated traffic flows for a day.

\section{CONCLUSION}

The group-based adaptive traffic-signal control systems developed in this study can deal with the systemic tendencies and transient fluctuations in traffic flows by integrating a global-optimization method with local adjustments of green signal time. The signal timings can be reactively adjusted with respect to transient fluctuations in traffic flows based on the cycle-structure. This development allows modifications to the signal sequence, cycle time, and duration of green time, as indicated by the cycle-by-cycle global proactive-optimization scheme. The key challenge is to identify the most appropriate cycle-structure to minimize the control delay in the cycle for a given predicted traffic condition and to implement the developed sub-modules using an adaptive traffic control logic, which is based on a group-based proactive global-optimization method of signal timings at the cycle level. The transient variations in traffic flows are captured and managed by the local reactive-control policy. To automatically adjust the current green time according to the pressure incurred by the current traffic conditions, a mathematical framework of the group-based maximum-pressure method is developed for the layout of a general signal-controlled intersection. A local clock for each signal group is provided to restrict the progression of each green time by imposing a transition zone, which is determined with reference to the group-based temporal variables.

In a series of test simulations, five models were examined under symmetric and asymmetric traffic conditions. These models, which comprised a fixed-time signal schedule, an actuated-signal control, and the several models developed in this study, were tested under various asymmetric conditions of traffic volume and turning proportions. The results of the computer simulations show that the integrated group-based adaptive traffic-signal control logic outperforms the other methods under diverse traffic conditions ranging from free flows to extreme congestion. Moreover, the performance of the proposed models under asymmetric traffic conditions is much better than that of the existing fixed-signal plan and the actuated-signal control. In addition, the proposed method performs well compared with the existing methods in the case study and the results confirm the applicability of the proposed models in the real world. The proposed methods are shown to be robust and effective, not only for optimizing signal schedules globally in each cycle to minimize the total delay, but also in simultaneously adjusting the current green phase according to the pressure on each signal group under diverse traffic conditions.

\section{Acknowledgements}


The work described in this paper was jointly supported by a Research Postgraduate Studentship and a grant from the University Research Committee of the University of Hong Kong (201411159005).

\section{References}

Coogan, S., Flores, C., Varaiya, P., 2017. Traffic predictive control from low-rank structure. Transportation Research Part B: Methodological 97, 1-22.

Heydecker, B.G., 1992. Sequencing of traffic signals. In Griffiths, J.D. (Ed.), Mathematics in Transport and Planning and Control. Clarendon Press, Oxford, 57-67.

Highway Capacity Manual, 2010. TRB, National Research Council, Washington D.C.

IMSL Problem-Solving Software Systems, 1989. User's manual, IMSL STAT/Library: FORTRAN subroutines for statistical analysis. IMSL, Inc., Houston, Tex. (2500 CityWest Blvd., Houston 77042-3020).

Lee, S., Wong, S.C., Li, Y.C., 2015a. Real-time estimation of lane-based queue lengths at isolated signalized junctions. Transportation Research Part C: Emerging Technologies 56, 1-17.

Lee, S., Wong, S.C., Pang, C.C.C., Choi, K., 2015b. Real-time estimation of lane-to-lane turning flows at isolated signalized junctions. Intelligent Transportation Systems, IEEE Transactions on $16,1549-1558$.

Lee, S., Wong, S.C., 2017a. Group-based approach to derivatives of delay models based on incremental queue accumulations for adaptive traffic control systems. Transportmetrica $B$, under review.

Lee, S., Wong, S.C., 2017b. Group-based approach to predictive delay model based on incremental queue accumulations for adaptive traffic control systems. Transportation Research Part B: Methodological 98, 1-20.

Lee, S., Wong, S.C., Varaiya, P., 2017. Group-based hierarchical adaptive traffic-signal control part I: Formulation. Transportation Research Part B: Methodological, under review.

Muralidharan, A., Coogan, S., Flores, C., Varaiya, P., 2016. Management of intersections with multi-modal high-resolution data. Transportation Research Part C: Emerging Technologies 68, 101-112.

Powell, M.J.D., 1989a. A tolerant algorithm for linearly constrained optimization calculations. DAMTP Report NA17, University of Cambridge, England.

Powell, M.J.D., 1989b. TOLMIN: A Fortran package for linearly constrained optimization calculations. DAMTP Report NA2, University of Cambridge, England.

Rogoff, P., Ayala, P., 2014. Revised departmental guidance on valuation of travel time in economic analysis. U.S. Department of Transportation, Washington D.C.

Santos, A., McGuckin, M., Nakamoto, H.Y., Gray, D., Liss, S., 2011. Summary of travel trends: 2009 national household travel survey. U.S. Department of Transportation, Washington D.C.

Transport Department, 1986. Road traffic signals 4, Transport Planning and Design Manual, Hong Kong Government.

Varaiya, P., 2013. Max pressure control of a network of signalized intersections. Transportation Research Part C: Emerging Technologies 36, 177-195.

Wong, S.C., 1995. Derivatives of the performance index for the traffic model from TRANSYT. Transportation Research Part B: Methodological 29, 303-327.

Wong, S.C., 1996. Group-based optimisation of signal timings using the TRANSYT traffic model. Transportation Research Part B: Methodological 30, 217-244.

Wong, S.C., 1997. Group-based optimisation of signal timings using parallel computing. Transportation Research Part C: Emerging Technologies 5, 123-139. 